\title{
Enhancing the Statistical Hybrid Model Performance in Overhead and Underground Medium Voltage Broadband over Power Lines Channels by Adopting Empirical Channel Attenuation Statistical Distribution
}

\author{
Athanasios G. Lazaropoulos* \\ School of Electrical and Computer Engineering / National Technical University of Athens / \\ 9 Iroon Polytechniou Street / Zografou, GR 15780
}

Received April 09, 2019; Accepted May 19, 2019; Published May 29, 2019

\begin{abstract}
Statistical hybrid model is a statistical channel model suitable for the broadband over power lines (BPL) networks while it is based on the statistical processing of channel attenuation and capacity values of preassumed BPL topology classes. One of the key operation elements of the statistical hybrid model, which affects its results fidelity, is the selection of the appropriate channel attenuation statistical distribution among a set of well-known channel attenuation statistical distributions (i.e., such as Gaussian, Lognormal, Wald, Weibull and Gumbel distributions). The selection of the appropriate channel attenuation statistical distribution becomes a hard task since it depends on a number of factors such as the power grid type -either overhead (OV) or underground (UN) power grid-, the representative distribution BPL topology of the examined class, the applied electromagnetic interference (EMI) policies and the used coupling scheme type. The contribution of this paper is to identify the conditions whether the Empirical channel attenuation statistical distribution can act as the default distribution of statistical hybrid model (modified statistical hybrid model) thus replacing the required comparison analysis prior to the selection of the aforementioned distributions of the initial statistical hybrid model. The evaluation comparison is based on the already applied metrics of capacity percentage change and average absolute capacity percentage change.
\end{abstract}

Keywords: Smart Grid; Broadband over Power Lines (BPL) networks; Power Line Communications (PLC); Distribution Power Grids; Capacity; Statistics; Modeling

\section{Introduction}

Living in the era of Internet of Things, we are witnessing an ever increasing number of devices with ubiquitous intelligence, which are interconnected via embedded systems and networks, that can communicate with humans and other devices changing our lives [1]. Under the auspices of the smart grid, further interoperability between electrical and electronic equipment can be delivered for the Internet of Things and, thus, new tremendous opportunities concerning the supported broadband applications of power utilities can be achieved [2]-[4]. Among the available communications solutions that have 
been proposed and are used across the smart grid, Broadband over Power Lines (BPL) technology can play an important role, since it may support an electronic communications channel (i.e., BPL channel) upon the already installed wired power grid infrastructure without adding extra complexity and cost in contrast with other communications solutions of the smart grid [4]-[16].

The initial statistical hybrid model that deals with the transmission and distribution BPL channel modeling through a statistical point of view has been demonstrated in [5], [6]. The core of the initial statistical hybrid model remains the deterministic well-validated hybrid model that has extensively been employed to examine the behavior of various multiconductor transmission line (MTL) configurations in transmission and distribution BPL networks [12]-[22]. Through the two interconnected submodules of the hybrid model, namely: (i) the bottom-up approach module; and (ii) the top-down approach module, the hybrid model acts as an internal phase of the statistical hybrid model flowchart that delivers as output crucial broadband performance metrics such as the channel attenuation and the capacity for given BPL topology, MTL configuration, coupling scheme, electromagnetic interference (EMI) policy and noise level. Actually, the initial statistical hybrid model consists of six phases where a set of indicative distribution BPL topologies act as the representative topologies of a set of respective distribution BPL topology classes. The statistical analysis of the distribution BPL topology classes offers the capacity range of each distribution BPL topology class for given power grid type, EMI policy, noise level, coupling scheme and channel attenuation statistical distribution. Actually, each distribution BPL topology class is filled with statistically equivalent BPL topologies, which are generated by appropriately deploying a random number generator, that are characterized by the same maximum likelihood estimators (MLEs) with the representative topology of the examined class for given power grid type, coupling scheme and channel attenuation statistical distribution. In [5], [6], a number of five well-known channel attenuation statistical distributions of the communications literature, say, Gaussian, Lognormal, Wald, Weibull and Gumbel distributions, have been benchmarked since they are involved in the MLE estimation and the operation of the random number generator. In accordance with [6], Weibull and Wald channel attenuation statistical distributions perform the best capacity estimations in OV MV and UN MV power grid types regardless of the examined BPL topology class and the applied coupling scheme when EMI policies committed to the broadband character of BPL technology are adopted (e.g., FCC Part 15 of [23]). When EMI policies less protective to the BPL technology are adopted (e.g., German Reg TP NB30 and the BBC / NATO Proposal of [13], [24], [25]), a more complex situation occurs in terms of the most suitable channel attenuation statistical distribution for the capacity estimations while the number of unsuccessful estimations significantly increases in all the cases examined. In this paper, it is investigated the potential of using only one channel attenuation statistical distribution, say, the Empirical channel attenuation statistical distribution, instead of trying to identify the best channel attenuation statistical distribution by taking into consideration each time the current operation settings. In accordance with [26], [27], the Empirical channel attenuation statistical distribution, which is the distribution function associated with the Empirical measure of coupling scheme channel attenuation differences, exploits the existing coupling scheme channel attenuation data of the examined distribution BPL topology class by computing their cumulative density function (CDF) for given coupling scheme while the adoption of the self-channel attenuation statistical distribution affects the flowchart of the statistical 
hybrid model in the MLE computation module (Phase $\mathrm{C}$ of the statistical hybrid model) and the random number generator module (Phase D of the statistical hybrid model). In this paper, the initial hybrid statistical model performance after the adoption of the Empirical channel attenuation statistical distribution, which is hereafter denoted as modified statistical hybrid model, is compared against the best case of the scenarios presented in [6] that deal with the initial statistical hybrid model. The performance metrics are going to be used for the benchmark are the percentage capacity change and absolute percentage capacity change.

The rest of this paper is organized as follows: In Section II, the OV MV and UN MV MTL configurations with the indicative BPL topology classes are presented. Section III summarizes the basics of the statistical hybrid model as well as the required flowchart changes for the adoption of the Empirical channel attenuation statistical distribution. In Section IV, a small briefing is given regarding the required operation settings of the initial and modified statistical hybrid model, say EMI policies, noise levels and applied coupling schemes. Section V compares the modified statistical hybrid model performance against the initial statistical hybrid model one for a number of different scenarios concerning the operation settings.

\section{OV MV and UN MV MTL Configurations and BPL Topologies}

In this Section, OV MV and UN MV MTL configurations, which are used in this paper, are here presented while the topological characteristics of the indicative representative OV MV and UN MV BPL topologies of the respective BPL topology classes are also reported.

\subsection{OV MV and UN MV MTL Configurations}

Typical cases of OV MV and UN MV distribution lines are depicted in Figs. 1(a) and 1(b) of [5], respectively. With reference to these figures, the examined OV MV distribution line consists of three parallel non-insulated phase conductors $\left(n^{\text {OvMV }}=3\right)$ spaced by $\Delta^{\text {OvNV }}$ and hang at typical heights $h^{\text {OvNV }}$ above lossy ground while the examined UN MV distribution line is the three-phase sector-type PILC distribution-class cable $\left(8 / 10 \mathrm{kV}, 3 \times 95 \mathrm{~mm}^{2} \mathrm{Cu}\right.$, PILC) surrounded by the shield and the armor conductor while the whole UN MV MTL configuration is buried to $1 \mathrm{~m}$ depth in lossy ground. Due to the common UN grounding practices, the analysis in this UN MV MTL configuration is focused only on the inner conductor set of the three phases surrounded by the shield $\left(n^{\mathrm{UNNV}}=3\right)$. The exact dimensions of the OV MV and UN MV MTL configurations are given in [17] as well the applied grounding practices and the impact of the considered lossy ground on BPL signal propagation.

\subsection{Indicative OV MV and UN MV BPL Topologies of Respective Topology Classes}

With reference to Fig. 2 of [5], BPL networks are divided into cascaded BPL topologies. Each BPL topology is bounded by the transmitting and receiving end repeaters while different number of branches $k_{v} k=1_{v} \cdots_{v} N$, distribution cable lengths $L_{k}, k=1_{s} \cdots, N+1$ and branch lengths $L_{b k}, k=1_{s} \cdots, N$ are encountered across the 
BPL signal transmission path. In accordance with [12]-[18], hybrid model treats each BPL topology separately as cascaded network modules.

With reference to Fig. 2 of [5], five indicative OV MV and UN MV BPL topologies are reported in Table 1 and 2, respectively. These indicative distribution

Table 1

Indicative OV MV BPL Topologies and Respective BPL Topology Classes [5], [28]

\begin{tabular}{|c|c|c|c|c|c|}
\hline $\begin{array}{c}\text { Topology } \\
\text { Number } \\
(\text { OV MV } \boldsymbol{l} \text { ) }\end{array}$ & $\begin{array}{c}\text { Indicative BPL } \\
\text { Topology Name }\end{array}$ & $\begin{array}{c}\text { BPL Topology Class } \\
\text { Description }\end{array}$ & $\begin{array}{c}\text { Number } \\
\text { of } \\
\text { Branches }\end{array}$ & $\begin{array}{c}\text { Length of Distribution } \\
\text { Lines }\end{array}$ & Length of Branching Lines \\
\hline OV MV 1 & Urban case A & $\begin{array}{c}\text { Typical OV MV BPL urban } \\
\text { topology class }\end{array}$ & 3 & $\begin{array}{c}L_{1}=500 \mathrm{~m}, L_{2}=200 \mathrm{~m}, \\
L_{3}=100 \mathrm{~m}, L_{4}=200 \mathrm{~m}\end{array}$ & $L_{\mathrm{b} 1=8 \mathrm{~m}, L_{\mathrm{b} 2}=13 \mathrm{~m}, L_{\mathrm{b} 3}=10 \mathrm{~m}}$ \\
\hline OV MV 2 & Urban case B & $\begin{array}{c}\text { Aggravated OV MV BPL } \\
\text { urban topology class }\end{array}$ & 5 & $\begin{array}{c}L_{1}=200 \mathrm{~m}, L_{2}=50 \mathrm{~m}, \\
L_{3}=100 \mathrm{~m}, L_{4}=200 \mathrm{~m}, \\
L_{5}=300 \mathrm{~m}, L_{6}=150 \mathrm{~m}\end{array}$ & $\begin{array}{c}L_{\mathrm{b} 1}=12 \mathrm{~m}, L_{\mathrm{b} 2}=5 \mathrm{~m}, L_{\mathrm{b} 3}=28 \mathrm{~m}, \\
L_{\mathrm{b} 4}=41 \mathrm{~m}, L_{\mathrm{b} 5}=17 \mathrm{~m}\end{array}$ \\
\hline OV MV 3 & Suburban case & $\begin{array}{c}\text { OV MV BPL suburban } \\
\text { topology class }\end{array}$ & 2 & $\begin{array}{c}L_{1}=500 \mathrm{~m}, L_{2}=400 \mathrm{~m}, \\
\mathrm{~L}_{3}=100 \mathrm{~m}\end{array}$ & $L_{\mathrm{b} 1}=50 \mathrm{~m}, L_{\mathrm{b} 2}=10 \mathrm{~m}$ \\
\hline OV MV 4 & Rural case & $\begin{array}{c}\text { OV MV BPL rural topology } \\
\text { class }\end{array}$ & 1 & $L_{1}=600 \mathrm{~m}, L_{2}=400 \mathrm{~m}$ & $L_{\mathrm{b} 1}=300 \mathrm{~m}$ \\
\hline OV MV 5 & "LOS" case & $\begin{array}{c}\text { OV MV BPL Line-of-Sight } \\
\text { transmission class }\end{array}$ & 0 & $L_{1}=1000 \mathrm{~m}$ & \\
\hline
\end{tabular}

Table 2

Indicative UN MV BPL Topologies and Respective BPL Topology Classes [5], [28]

\begin{tabular}{|c|c|c|c|c|c|}
\hline $\begin{array}{c}\text { Topology } \\
\text { Number } \\
(\text { UN MV } l \text { ) }\end{array}$ & $\begin{array}{c}\text { Indicative BPL } \\
\text { Topology Name }\end{array}$ & $\begin{array}{c}\text { BPL Topology Class } \\
\text { Description }\end{array}$ & $\begin{array}{c}\text { Number } \\
\text { of } \\
\text { Branches }\end{array}$ & $\begin{array}{c}\text { Length of Distribution } \\
\text { Lines }\end{array}$ & Length of Branching Lines \\
\hline UN MV 1 & Urban case A & $\begin{array}{c}\text { Typical UN MV BPL urban } \\
\text { topology class }\end{array}$ & 3 & $\begin{array}{c}L_{1}=70 \mathrm{~m}, L_{2}=55 \mathrm{~m}, \\
L_{3}=45 \mathrm{~m}, L_{4}=30 \mathrm{~m}\end{array}$ & $L_{\mathrm{b} 1}=12 \mathrm{~m}, L_{\mathrm{b} 2}=7 \mathrm{~m}, L_{\mathrm{b} 3}=21 \mathrm{~m}$ \\
\hline UN MV 2 & Urban case B & $\begin{array}{c}\text { Aggravated UN MV BPL } \\
\text { urban topology class }\end{array}$ & 5 & $\begin{array}{c}L_{1}=40 \mathrm{~m}, L_{2}=10 \mathrm{~m}, \\
L_{3}=20 \mathrm{~m}, L_{4}=40 \mathrm{~m}, \\
L_{5}=60 \mathrm{~m}, L_{6}=30 \mathrm{~m}\end{array}$ & $\begin{array}{c}L_{\mathrm{b} 1}=22 \mathrm{~m}, L_{\mathrm{b} 2}=12 \mathrm{~m}, L_{\mathrm{b} 3}=8 \mathrm{~m}, \\
L_{\mathrm{b} 4}=2 \mathrm{~m}, L_{\mathrm{b} 5}=17 \mathrm{~m}\end{array}$ \\
\hline UN MV 3 & Suburban case & $\begin{array}{c}\text { UN MV BPL suburban } \\
\text { topology class }\end{array}$ & 2 & $\begin{array}{c}L_{1}=50 \mathrm{~m}, L_{2}=100 \mathrm{~m}, \\
L_{3}=50 \mathrm{~m}\end{array}$ & $L_{\mathrm{b} 1}=60 \mathrm{~m}, L_{\mathrm{b} 2}=30 \mathrm{~m}$ \\
\hline UN MV 4 & Rural case & $\begin{array}{c}\text { UN MV BPL rural topology } \\
\text { class }\end{array}$ & 1 & $L_{1}=50 \mathrm{~m}, L_{2}=150 \mathrm{~m}$ & $L_{\mathrm{b} 1}=100 \mathrm{~m}$ \\
\hline UN MV 5 & "LOS" case & $\begin{array}{c}\text { UN MV BPL "LOS" } \\
\text { transmission class }\end{array}$ & 0 & $L_{1}=200 \mathrm{~m}$ & \\
\hline
\end{tabular}

BPL topologies act as the representative BPL topologies of respective BPL topology classes. Note that average long end-to-end connections of $1000 \mathrm{~m}$ and $200 \mathrm{~m}$ are assumed for the indicative OV MV and UN MV BPL topologies, respectively.

\section{Initial and Modified Statistical Hybrid Model}

In this Section, the flowchart of the initial statistical hybrid model, which exploits MLEs of the five channel attenuation statistical distributions, and the phases involved are presented with respect to [5], [6]. Then, the required changes in the flowchart and the 
new phases of the modified statistical hybrid model after the replacement of the five channel attenuation statistical distributions of the initial statistical hybrid model from the Empirical channel attenuation statistical distribution are defined as well as the Empirical channel attenuation statistical distribution itself.

\subsection{Statistical Hybrid Model with MLEs (Initial Statistical Hybrid Model)}

The initial statistical hybrid model, which exploits the five well-known channel attenuation statistical distributions (say, Gaussian, Lognormal, Wald, Weibull and Gumbel distributions), has been detailed and numerically validated in [5], [6]. In Fig. 1(a), the flowchart of the initial statistical hybrid model is given in terms of a business process model notification (BPMN) diagram.

In accordance with [5] and with respect to Fig. 1(a), the initial statistical hybrid model consists of six phases, namely: (i) Phase A. The hybrid model takes as inputs the distribution power grid type, the indicative distribution BPL topology, the respective distribution MTL configuration and the applied coupling scheme while it gives as output the coupling scheme channel transfer function $1 \times Q$ line vector $\mathrm{H}_{\mathbb{l}, 1}^{\mathrm{GC}}(\mathbf{f})$ where $Q$ is the number of flat-fading subchannels in the examined frequency range, []$^{\mathrm{G}}$ denotes the examined distribution power grid type (i.e., OV MV or UN MV), [. $]^{c}$ denotes the applied coupling scheme (see Sec.3.2 of [5]), $\mathbf{f}$ is the $1 \times Q$ line vector that consists of the flat-fading subchannel start frequencies $f_{q}, q=1_{, \ldots}, Q$ and $l$ is the topology number (see Table 1 and 2). (ii) Phase B. The coupling scheme channel attenuation difference module $\Delta$ of this Phase receives as input the coupling scheme channel transfer function line vector from Phase A and gives as output the coupling scheme channel attenuation difference $\Delta \mathbf{A}_{\mathbb{1}, 1}^{\mathrm{G} C}(\mathrm{f})$ between each indicative distribution BPL topology and its respective "LOS" case for given power grid type and BPL topology class. (iii) Phase $C$. MLE computation module receives as input the coupling scheme channel attenuation difference and gives as output the MLEs for each of the five channel attenuation statistical distributions for given distribution BPL topology and coupling scheme. Note that this Phase is a synonym of the application of the five channel attenuation statistical distribution and the business process reengineering of this paper is focused on this Phase. To symbolize the existence of different MLE sets, three small vertical lines are applied to the output files of Phases C-F in Fig. 1(a). (iv) Phase $D$. The random number generator receives as input the MLEs of each channel attenuation statistical distribution and gives as output the random number $1 \times Q$ line vector $\mathbf{R}_{l, p}^{\mathrm{G}, \mathrm{C}, \mathrm{D}}$ for given power grid type, coupling scheme and indicative distribution BPL topology where []$^{\mathrm{D}}$ denotes the applied channel attenuation statistical distribution, $p, p=1, \ldots, P+1$ is the member number in the BPL topology class and $P$ is the member number of each class. (v) Phase E. $\Delta^{-1}$ module performs the inverse procedure of $\Delta$ module of Phase $\mathrm{B}$ since it takes as input the random number line vector for given power grid type, coupling scheme, indicative distribution BPL topology and channel attenuation statistical distribution and gives as output the coupling scheme channel transfer function line vector $\mathrm{H}_{l, p}^{\mathrm{G}, \mathrm{C}, \mathrm{D}}(\mathrm{f})$ of each of the $P$ members of each BPL topology class for given power grid type, coupling scheme and channel attenuation statistical distribution. After Phase E, each distribution BPL topology class consists of $P+1$ members whose coupling scheme channel transfer functions for given power grid type, coupling scheme and channel attenuation statistical distribution are considered as the output of the Phase E. (vi) Phase F. This Phase receives as input the coupling scheme 
channel transfer functions of each distribution BPL topology class for given power grid type, coupling scheme and channel attenuation statistical distribution and gives as output

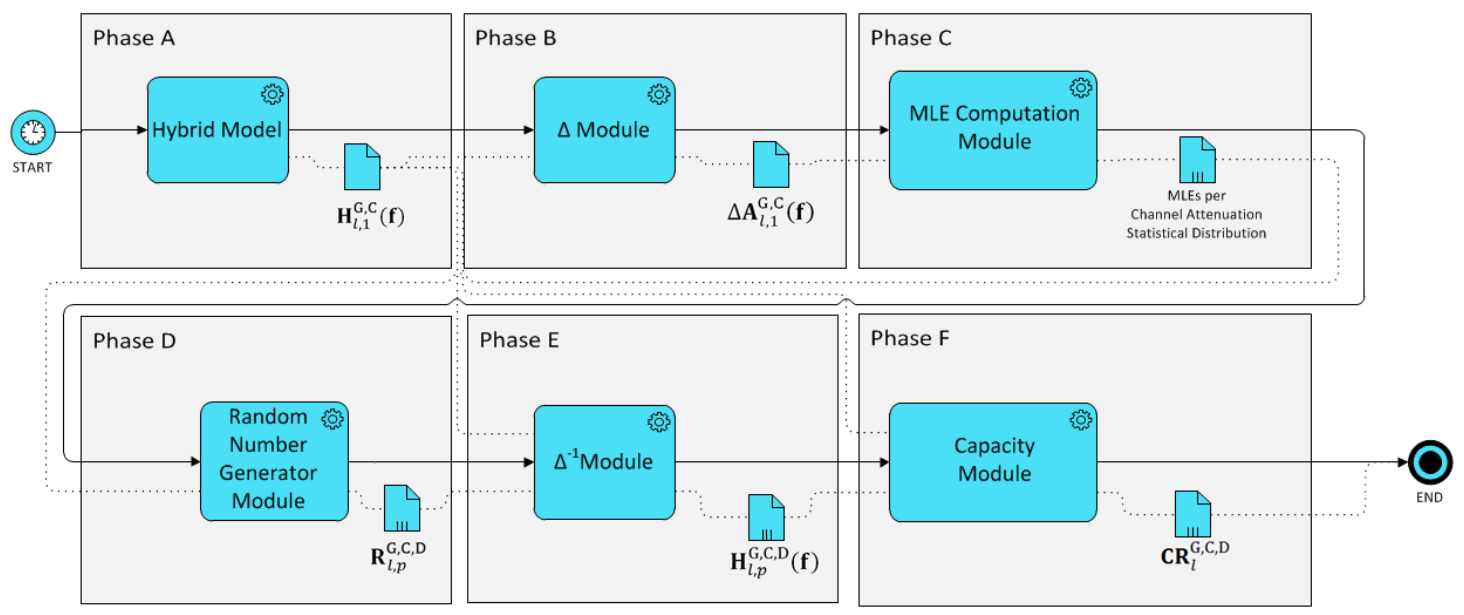

(a)

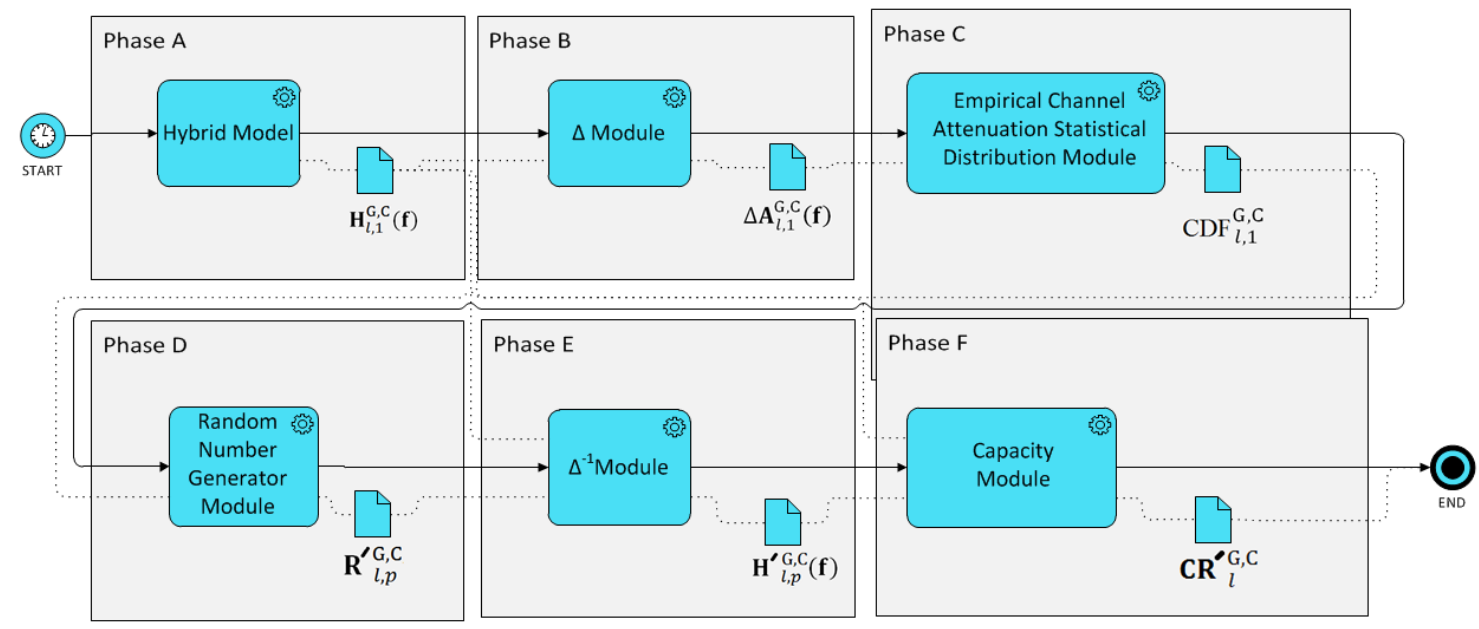

(b)

Fig. 1. Business Process Reengineering of the Statistical Hybrid Model. (a) BPMN diagram of the Initial Statistical Hybrid Model [5]. (b) BPMN diagram of the Modified Statistical Hybrid Model.

the capacity range of each distribution BPL topology class

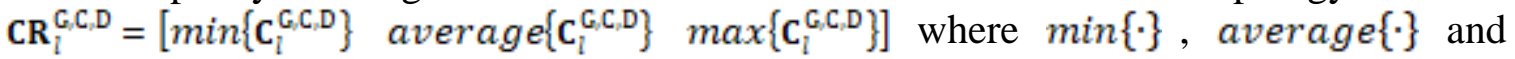
$\max \{x\}$ computes the minimum, the average and the maximum value of distribution BPL topology class capacity $\mathrm{C}_{\mathbb{I}}^{\mathrm{GC} D}$ that consists of all the capacities of its $P+1$ members.

In accordance with [6], the results of the initial statistical hybrid model are benchmarked in terms of the capacity percentage change and the average absolute capacity percentage change with respect to the applied channel attenuation statistical distribution, namely the Gaussian, Lognormal, Wald, Weibull and Gumbel distribution. A capacity estimation can be considered as successful when: (i) the capacity range of each distribution BPL topology class comprises the capacity of the respective indicative distribution BPL topology; and (ii) the average capacity value remains very close to the respective one of the indicative distribution BPL topology. In [6], it has been 
demonstrated that Weibull and Wald channel attenuation statistical distributions perform the best maximum likelihood estimations in OV MV and UN MV power grid types, respectively, regardless of the examined BPL topology class and the applied coupling scheme for EMI policies of high injected power spectral density (IPSD) limits such as those of FCC Part 15 (see Sec.4.2). As the IPSD limits become lower, a mixed scenario regarding the selection of the most suitable channel attenuation statistical distribution for the capacity estimation among the BPL topology classes and power grid types occurs.

\subsection{The Empirical Channel Attenuation Statistical Distribution of the Modified Statistical Hybrid Model}

The insertion of the Empirical channel attenuation statistical distribution aims at bypassing the difficult task of the evaluation and the selection among the available channel attenuation statistical distributions that are based on MLEs. In Fig. 1(b), the flowchart of the modified statistical hybrid model is given in terms of a BPMN diagram thus allowing the process comparison between the initial and the modified statistical hybrid models.

By comparing Figs. 1(a) and 1(b), the following observations concerning the operation of the modified statistical hybrid model can be made, namely:

- Phases A and B remain identical between the initial and modified statistical hybrid model.

- The insertion of the Empirical channel attenuation statistical distribution mainly affects Phases C and D. More specifically, the following changes occur for the Phases $\mathrm{C}$ and $\mathrm{D}$ :

- Phase $C$. Instead of the MLE computation module, the Empirical channel attenuation statistical distribution module is here added that receives as input the coupling scheme channel attenuation difference while it gives as output the Empirical CDF of the coupling scheme channel attenuation difference $\mathrm{CDF}_{l_{3}, 1}^{\mathrm{G} C}$ for given distribution BPL topology and coupling scheme. Note that each coupling scheme channel attenuation difference of the indicative distribution BPL topologies is characterized by its own CDF and, for this reason, the term of Empirical channel attenuation statistical distribution is used to describe this unique statistical distribution behaviour [29].

- Phase D. Conversely to the initial statistical hybrid model, the random number generator receives as input the Empirical CDF of the examined coupling scheme channel attenuation difference instead of MLEs. With reference to [26], [27], random number generator module performs an inverse interpolation to achieve CDF projection of the random values thus giving as output the random number $1 \times Q$ line vector $\mathbf{R}_{b_{j},}^{\mathbf{G}_{;} \mathbf{C}}$ for given coupling scheme and indicative distribution BPL topology. Actually, given the MLE sets, the random number generator module of the initial statistical hybrid model computes the corresponding continuous CDF as reported in Appendix A of [5] for each of the five channel attenuation statistical distribution and then the reverse interpolation is easily applied to these continuous CDFs. In contrast, an additional interpolation for the 
discrete CDF of the modified hybrid statistical model may be required if the random number is not among the available discrete CDF values.

- Phases E and F are slightly affected since these two Phases now operate with only one statistical distribution, say the Empirical channel attenuation statistical distribution, instead of the five ones of the initial statistical hybrid model.

- The output of the modified statistical hybrid model, which coincides with the output of the Phase $\mathrm{F}$, is the capacity range of each distribution BPL topology

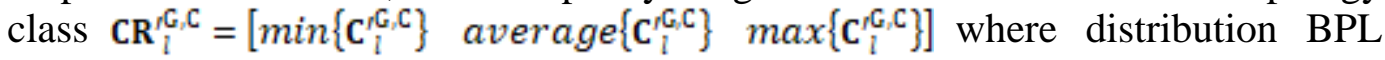
topology class capacity $\mathrm{C}_{\mathbb{I}}^{\mathrm{C} C}$ consists of all the capacities of its $P+1$ members when the Empirical channel attenuation statistical distribution is applied.

Similarly to the benchmark of the initial statistical hybrid model, the performance results of the modified statistical hybrid model are going to be investigated by the application of the metrics of capacity percentage change and average absolute capacity percentage change for the set of scenarios presented in [6]. In fact, each scenario is characterized by its own operation settings on the basis of a default operation setting set presented in Sec.4.

\section{Operation Settings of Statistical Hybrid Model}

In this Section, the default operation settings of the statistical hybrid model, which remain common for the initial and the modified model and constitute the baseline scenario, are presented. Also, the set of scenarios of [6], which is here deployed in order to assess the performance of the initial and modified statistical hybrid model performance comparison, is detailed as well as the respective operation settings.

\subsection{Primary and Secondary Operation Settings}

As already identified in [6], the distribution power grid type, the BPL topology class, the channel attenuation statistical distribution, EMI policy, noise level and applied coupling scheme are the primary factors that influence the performance of the initial statistical hybrid model. The same primary factors are also going to influence the modified statistical hybrid model performance apart from the channel attenuation statistical distributions since the Empirical channel attenuation statistical distribution is used in the latter model case to substitute them. As EMI policies, noise levels and applied coupling schemes are concerned, there is a selection of default values (see Secs.4.2, 4.3 and 4.4, respectively) that constitutes the default operation settings while the value variation of the aforementioned three primary factors constitute the different scenarios that are examined in the following numerical analysis.

Except for the primary operation settings, there is a number of secondary operation settings that are required by both the initial and the modified statistical hybrid model for their fine operation, namely:

- Circuital parameters of the hybrid model. To apply the hybrid model of Phase A of the statistical hybrid model, the set of circuital parameters, which is detailed in [7]-[9], [11]-[17], [19], [20], [22], [30]-[41], needs to be assumed, synoptically:

$\circ$ The identicalness of the branching and the distribution cables.

o The full activation of the interconnections between the distribution and branch conductors. 
$\circ$ The matched termination in the transmitting and receiving ends.

○ The open-circuit branch terminations.

- Representative distribution BPL topology of each topology class. With reference to Tables 1 and 2, the indicative OV MV and UN MV BPL topologies are assumed to be the representative ones for the respective OV MV and UN MV BPL topology classes. The OV MV and UN MV MTL configurations, which are used in this paper, are presented in Fig. 1(a) and 1(b) of [5], respectively.

- Number of members of each distribution BPL topology class. 100 member distribution BPL topologies (i.e., $P=100$ ) are assumed to be added in each BPL topology class of Tables 1 and 2.

- Frequency range and flat-fading subchannel frequency spacing. The BPL operation frequency range and the flat-fading subchannel frequency spacing are assumed to be equal to $3-30 \mathrm{MHz}$ and $0.1 \mathrm{MHz}$ (i.e, $f_{s}=0.1 \mathrm{MHz}$ ), respectively. Hence, there are 270 subchannels in the frequency range of interest (i.e., $Q=270$ ).

- Negative and zero values of the coupling scheme channel attenuation differences. During the computation of the coupling scheme channel attenuation differences in the Phase B of [5], values that are greater or equal to zero are expected in the vast majority of the cases. However, in the scarce cases of negative coupling scheme channel attenuation differences and in "LOS" cases, the coupling scheme channel attenuation differences are assumed to be equal to an arbitrarily low value, say $1 \times 10^{-11}$. Since the MLE computation of Lognormal, Wald and Weibull channel attenuation distributions comprises natural logarithms and denominators, this low value is used in order not to render the aforementioned terms infinite in the case of the initial statistical hybrid model. For comparison reasons between the initial and modified statistical hybrid model and in accordance with [5], [6], the same assumption remains valid for the modified statistical hybrid model.

\subsection{EMI Policies and Default Operation Settings}

BPL networks and systems operate in frequency ranges that are already occupied by other licensed communications services. Since, BPL networks and systems are, at the same time, unintentional EMI transmitters to these already licensed communications services (e.g., aeronautical radionavigation, radio astronomy, mobile satellite and maritime mobile) and EMI receivers from the aforementioned services, EMI policies that try to regulate these EMI emissions have been proposed by a number of regulation bodies. These EMI policies may correspond to respective IPSD limits $p(\cdot)$ that regulate the EMI emissions of BPL networks and systems so that BPL networks and systems do not interfere with the other already licensed communications services in the same frequency band of operation.

Among the proposals concerning EMI policies, the most noted are FCC Part 15, German Reg TP NB30 and the BBC / NATO Proposal. The electric field strength limits proposed by the above proposals are presented in [43], [44], [112] while the respective IPSD limits are determined in [13], [15], [25], [44]. During the following analysis, FCC Part 15 defines the default primary operation setting concerning EMI policy while German Reg TP NB30 and the BBC / NATO Proposal are the different scenarios concerning the EMI policy. 


\subsection{Noise and Default Operation Settings}

In accordance with Sec. 4.3 of [5], capacity of distribution BPL networks depends on the applied MTL configuration, the examined BPL topology, the coupling scheme applied, the EMI policies adopted and the noise environment [7]-[14]. Actually, with reference to eq. (3) of [5], noise can be identified as a leading inherent BPL deficiency that critically degrades the BPL network capacities [45]. However, a typical noise scenario for capacity computations is the adoption of uniform AWGN PSD levels that can be considered as a very accurate approximation during the capacity computations as presented through FL noise model in [45], [46]. Therefore, as the default operation properties of noise properties in distribution BPL networks, $-105 \mathrm{dBm} / \mathrm{Hz}$ and $-135 \mathrm{dBm} / \mathrm{Hz}$ are the default AWGN PSD limit levels $N(\cdot)$ for OV MV and UN MV BPL networks, respectively, in the $3-30 \mathrm{MHz}$ frequency range are considered. Note that only default noise operation settings are assumed without other noise scenarios.

\subsection{Coupling Schemes and Default Operation Settings}

BPL signals are injected into and extracted from the lines of OV MV and UN MV BPL networks through different coupling schemes that are implemented by coupling scheme modules (CS modules) [11], [17], [19], [28], [47]. CS modules are integrated into the deterministic hybrid model of Phase A of the initial and modified statistical hybrid model. In accordance with [28], [47], CS2 module that is applied in this paper and remains the most recently upgraded coupling CS module for BPL networks may support three types of coupling schemes, namely: (1) Coupling Scheme Type 1: Wire-to-Ground $(W t G)$ or Shield-to-Phase $(S t P)$ coupling schemes for OV or UN BPL networks, respectively; (2) Coupling Scheme Type 2: Wire-to-Wire (WtW) or Phase-to-Phase (PtP) coupling schemes for OV or UN BPL networks, respectively; and (3) Coupling Scheme Type 3: MultiWire-to-MultiWire (MtM) or MultiPhase-to-MultiPhase (MtM) coupling schemes for OV or UN BPL networks, respectively. Depending on the involved conductors of the examined MTL configuration and the power restrictions of [28], [47] concerning the power allocation among the involved conductors, different coupling schemes can occur for given coupling scheme type. In accordance to [6], to give a broader image of the coupling scheme selection impact, coupling scheme types 1,2 and 3 are examined in this paper, namely:

- Coupling Scheme Type 1. $\mathrm{WtG}^{1}$ and $\mathrm{StP}^{1}$ coupling schemes are deployed for the assessment of OV MV and UN MV BPL topology classes, respectively.

- Coupling Scheme Type 2. WtW $\mathrm{W}^{1-2}$ and $\mathrm{PtP}^{1-2}$ coupling schemes are deployed for the assessment of OV MV and UN MV BPL topology classes, respectively.

- Coupling Scheme Type 3. $\mathrm{MtM}_{0.8-0.1-0.1}^{1-2-3}$ coupling scheme is deployed for the assessment of both OV MV and UN MV BPL topology classes.

In accordance with [6], the two coupling schemes of type 1 are assumed to be the default ones while the coupling schemes of type 2 and 3 define the different coupling scheme type scenarios.

\section{Numerical Results and Discussion}

In this Section, numerical results concerning the performance comparison of the initial and modified statistical hybrid model are presented. Taking into account the 
already identified default operation settings of Secs.4.1-4.4, the possibility of only adopting the Empirical channel attenuation statistical distribution of the modified statistical hybrid model against the five aforementioned channel attenuation statistical distributions of the initial statistical hybrid model is assessed. In fact, the comparison is based on the paper format of [6].

\subsection{Initial and Modified Statistical Hybrid Model - Coupling Scheme Channel Attenuation Differences and CDFs of the Indicative OV MV and UN MV BPL Topologies for the Default Operation Settings}

By comparing the flowcharts of the initial and modified statistical hybrid model of the respective Figs. 1(a) and 1(b), their main difference is located at the Phase $\mathrm{C}$ where MLE computation module and Empirical channel attenuation statistical distribution module are deployed for the initial and modified statistical hybrid model, respectively.

However, both modules receive as input the coupling scheme channel attenuation difference data for given indicative distribution BPL topology and coupling scheme. In Fig. 2, the coupling scheme channel attenuation difference of the indicative OV MV BPL topology of urban case A, which is reported in Table 1, is plotted versus the frequency in the $3-30 \mathrm{MHz}$ frequency band when $\mathrm{WtG}^{1}$ coupling scheme, which is anyway the default coupling scheme for OV MV BPL topologies, is applied. In the same figure, the plots of the indicative OV MV BPL topologies of urban case B, suburban case and rural case, which are also reported in Table 1, are shown. It should be reminded here that the "LOS" case of Table 1 is not presented in Fig. 2 since its coupling scheme channel attenuation differences are equal to zero. Note that the plots of the indicative OV MV BPL topologies are computed with reference to the OV MV BPL "LOS" case -see eq. (5) of [5]-. In Fig. 3, same curves with Fig. 2 are demonstrated but for the case of the indicative UN MV BPL topologies of Table 2 when $\mathrm{StP}^{1}$ coupling scheme is applied in the same operation frequency band.

Since the coupling scheme channel attenuation difference data are received by the MLE computation module and Empirical channel attenuation statistical distribution module, MLE computation module and Empirical channel attenuation statistical distribution module give as output the MLEs of the five statistical distribution and the Empirical CDF, respectively. In the case of the initial statistical hybrid model, the random number generator module of Phase D initially and internally computes CDFs of the five channel attenuation statistical distribution on the basis of the respective MLEs of Phase C. Hence, either initial or modified statistical hybrid model compute CDFs of the applied channel attenuation statistical distributions. With reference to Fig. 2, in Fig. 4(a), CDFs of the five channel attenuation statistical distributions (say, Gaussian, Lognormal, Wald, Weibull and Gumbel distributions) of the initial statistical hybrid model and of the Empirical channel attenuation statistical distribution of the modified statistical hybrid model are plotted versus the coupling scheme channel attenuation difference for the case of the OV MV BPL urban case A. In Figs. 4(b)-(d), same plots with Fig. 4(a) are given bur for the case of the OV MV BPL urban case B, suburban case and rural case, respectively. In Figs. 5(a)-(d), same curves are presented with Figs. 4(a)-(d) but for the case of the indicative UN MV BPL topologies. 


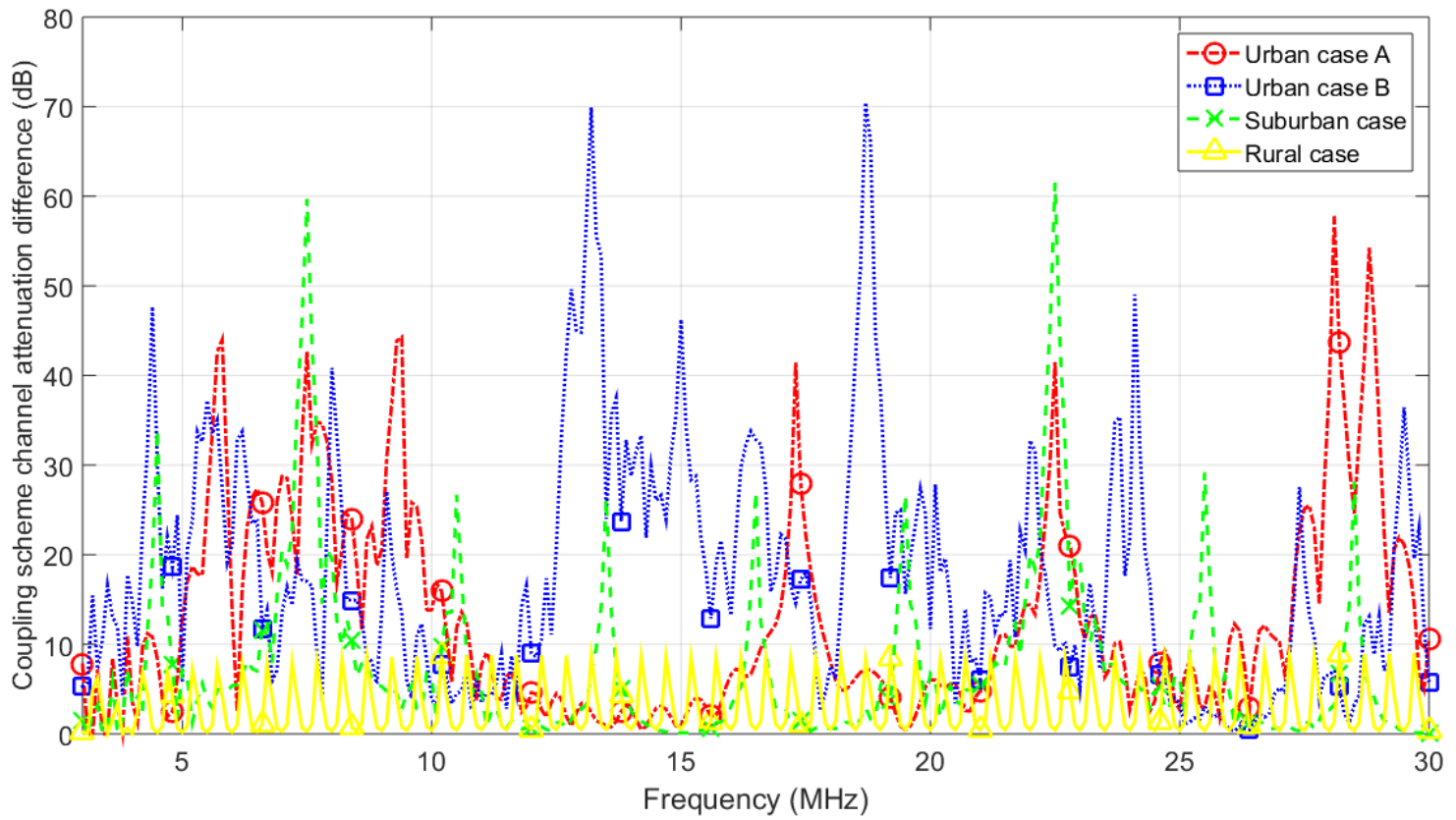

Fig. 2. Coupling scheme channel attenuation difference of the indicative OV MV BPL topologies of Table 1 in the $3-30 \mathrm{MHz}$ frequency band when $\mathrm{WtG}^{1}$ coupling scheme is deployed (the subchannel frequency spacing is equal to $0.1 \mathrm{MHz}$ ).

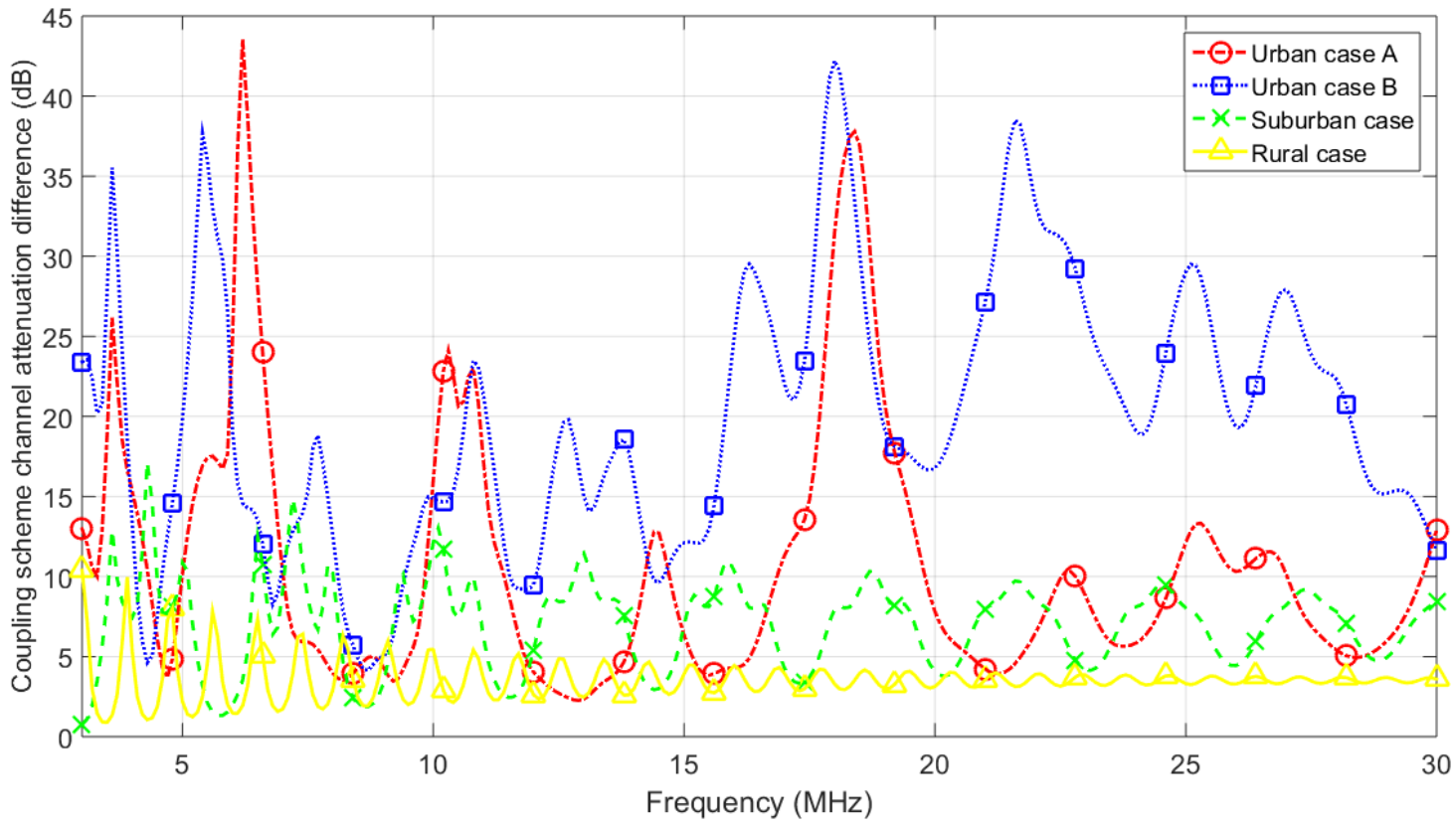

Fig. 3. Coupling scheme channel attenuation difference of the indicative UN MV BPL topologies of Table 2 in the $3-30 \mathrm{MHz}$ frequency band when $\mathrm{StP}^{1}$ coupling scheme is deployed (the subchannel frequency spacing is equal to $0.1 \mathrm{MHz}$ ). 


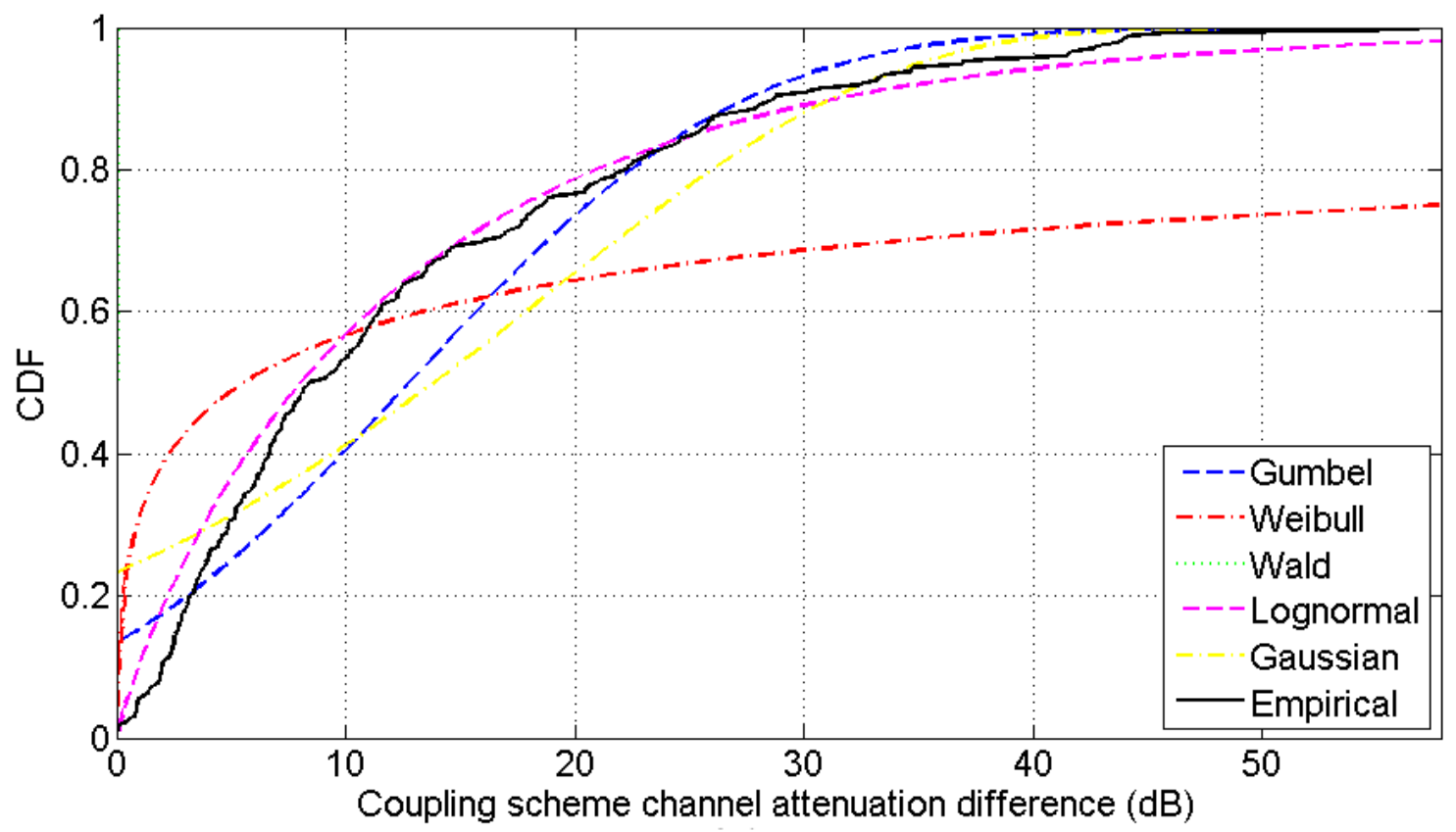

(a)

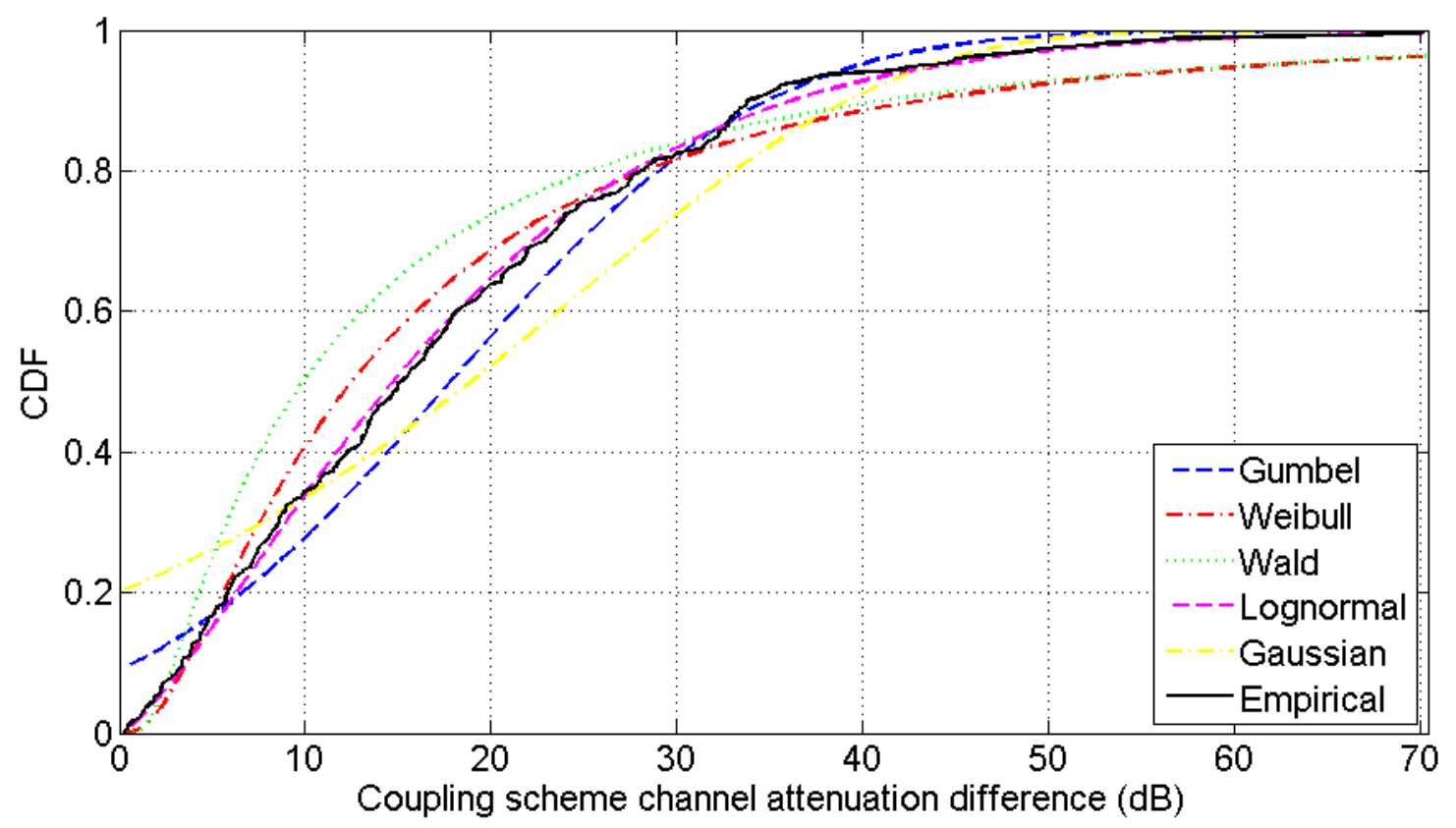

(b) 


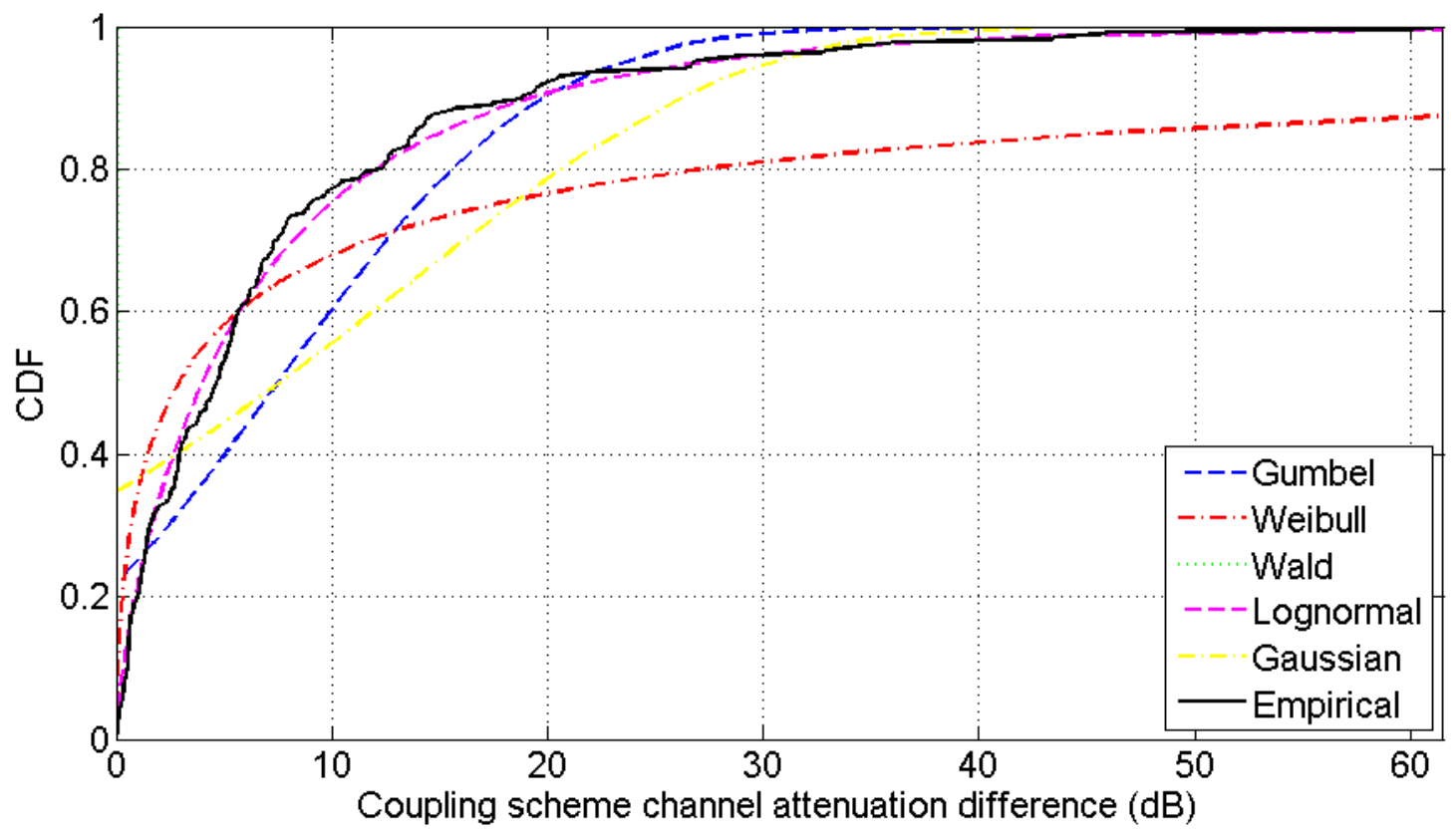

(c)

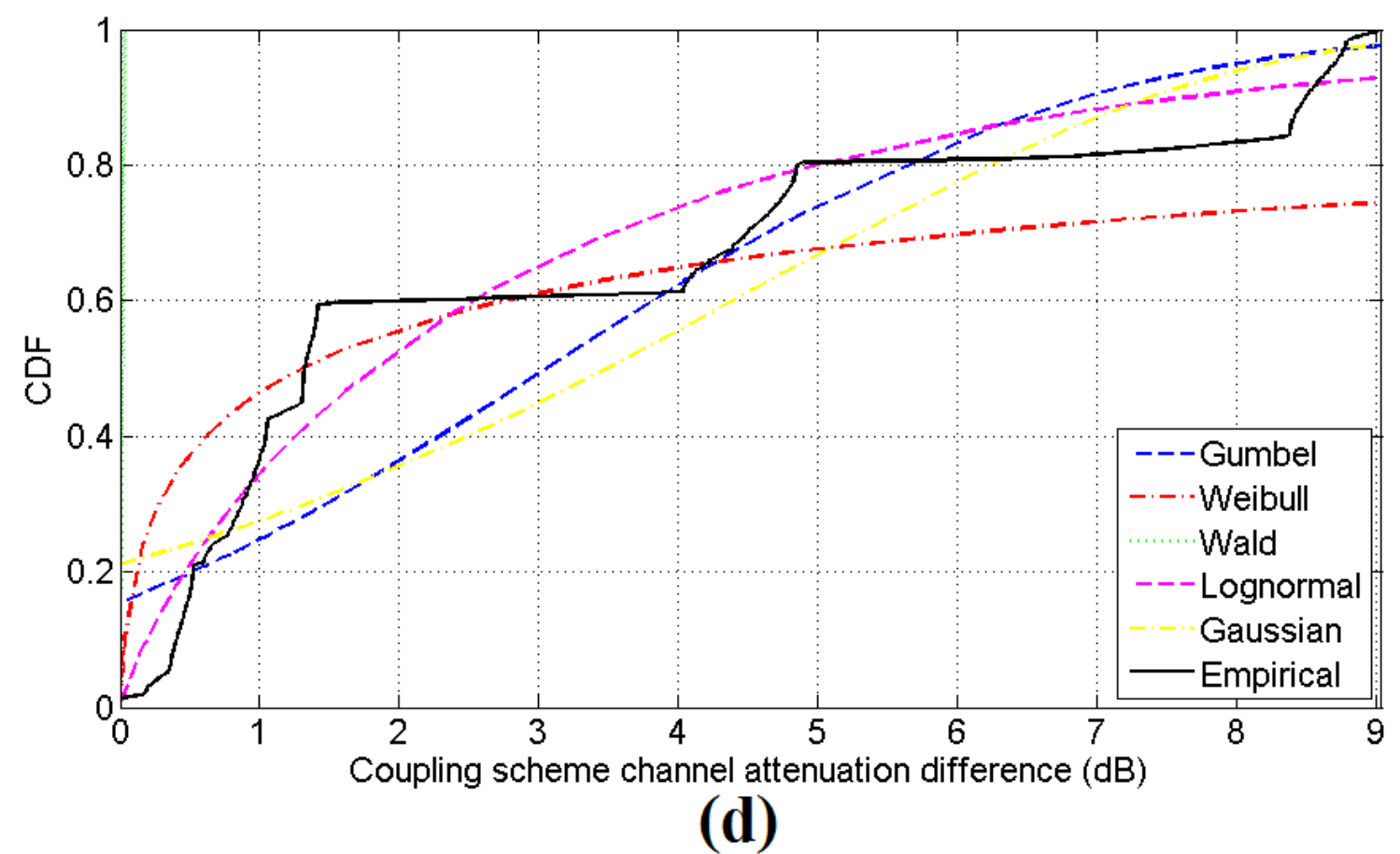

Fig. 4. CDF of the indicative OV MV BPL topologies of Table 1 versus coupling scheme channel attenuation difference in the $3-30 \mathrm{MHz}$ frequency band when $\mathrm{WtG}^{1}$ coupling scheme is deployed. (a) Urban case A. (b) Urban case B. (c) Suburban case. (d) Rural case. 


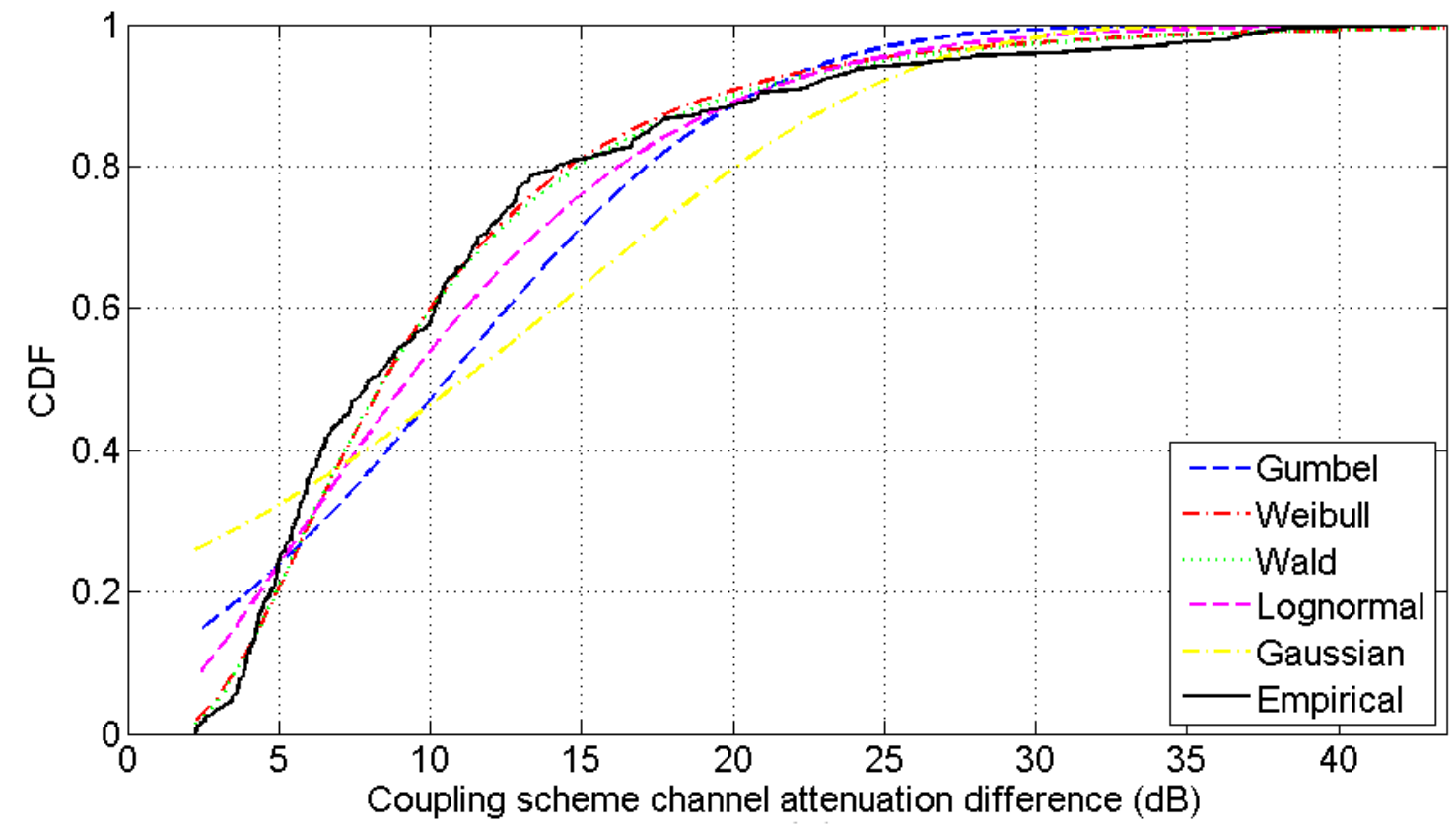

(a)

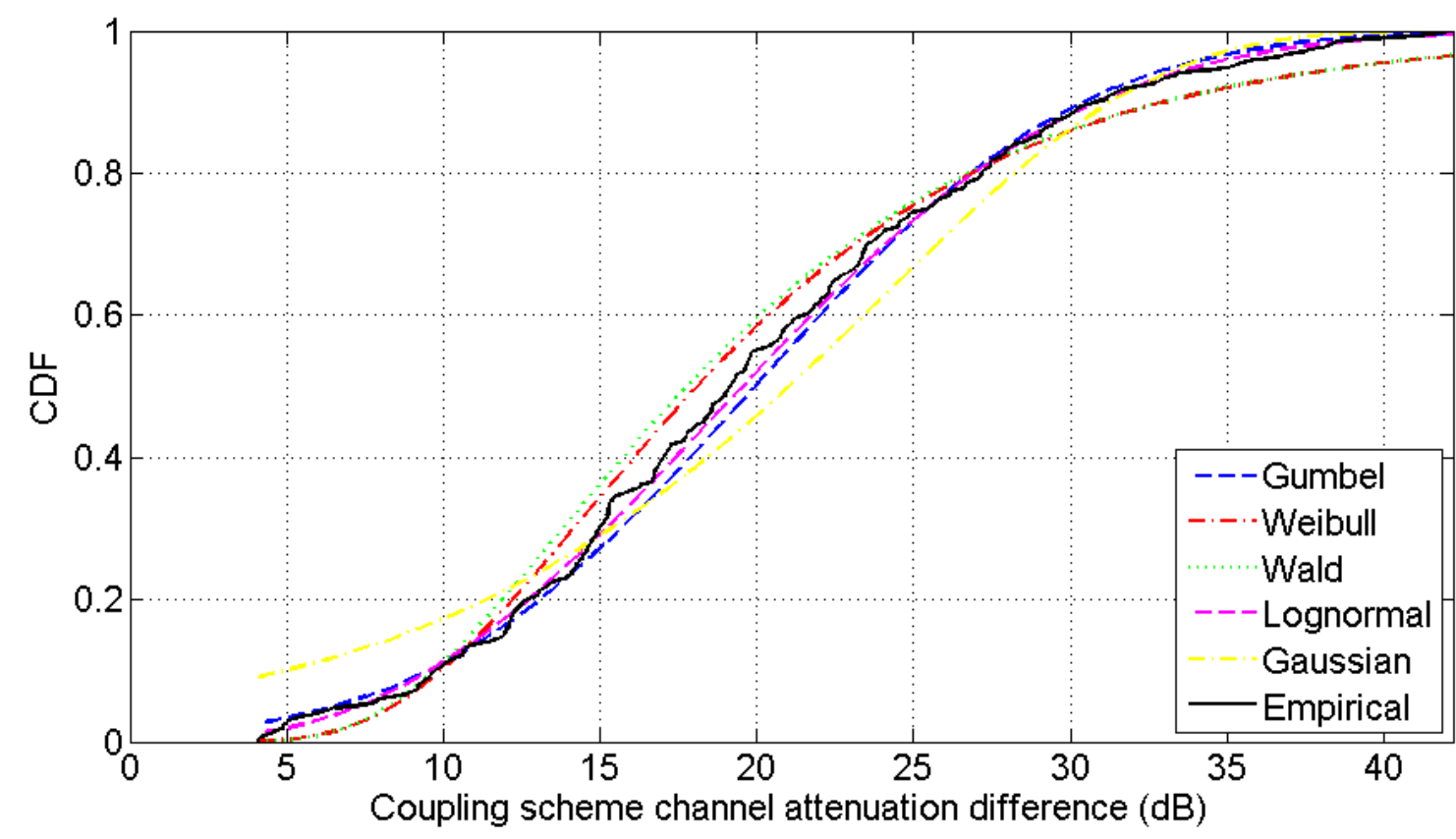

(b) 


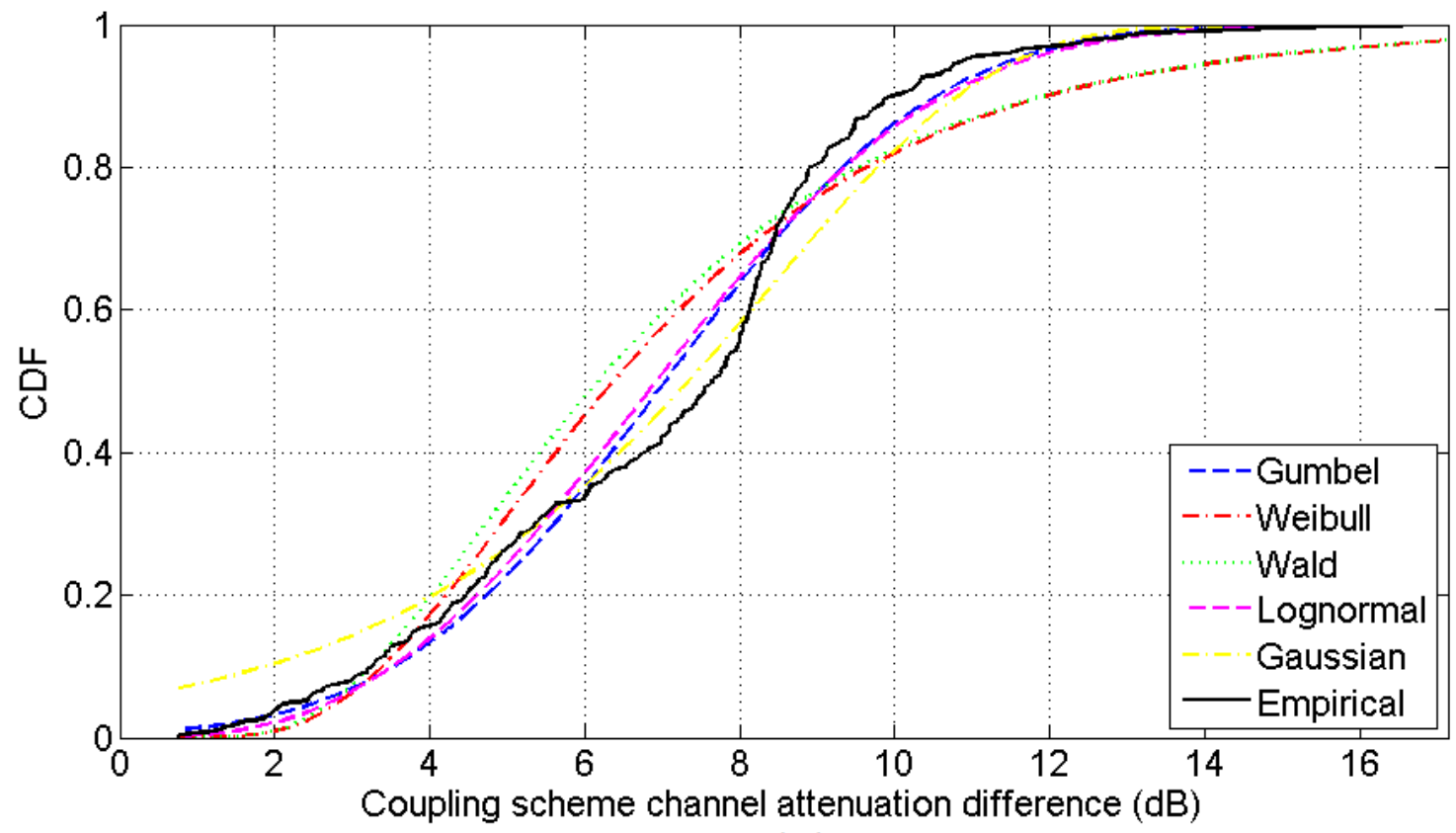

(c)

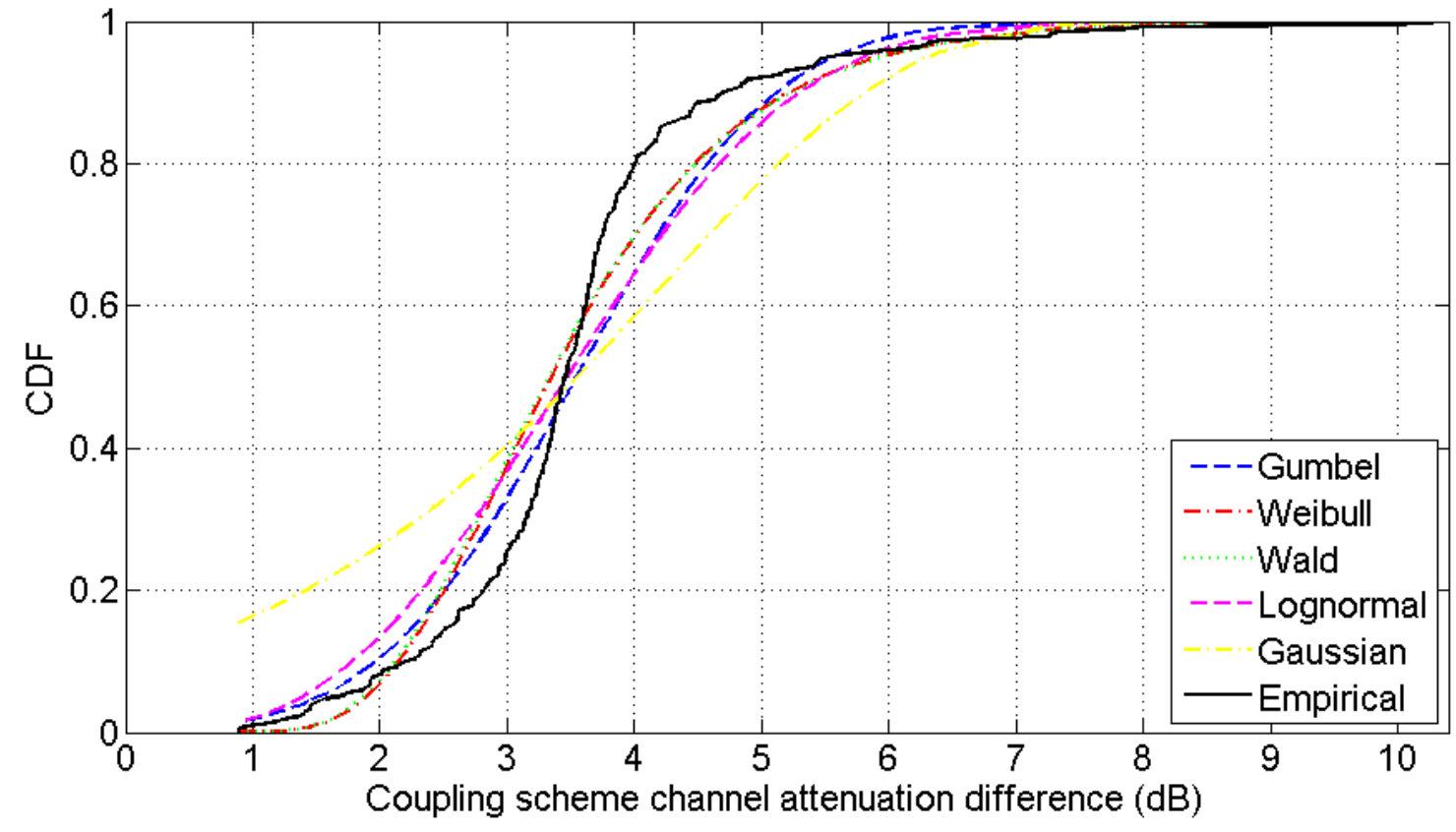

(d)

Fig. 5. Same plots with Fig. 4 but for the case of the indicative UN MV BPL topologies of Table 2 when $\mathrm{StP}^{1}$ coupling scheme is deployed.

From Figs. 2-5, several interesting remarks can be reported concerning the CDF behavior of the channel attenuation statistical distributions of the initial and modified statistical hybrid model. More specifically: 
- By comparing Figs. 2 and 3, it is evident that the coupling scheme channel attenuation difference of OV MV BPL topologies presents higher spectral notches in comparison with the ones of UN MV BPL topologies. This is due to the fact that the main channel attenuation mechanism of OV MV BPL networks is the multipath propagation in contrast with the high "LOS" propagation of UN MV BPL networks [8], [12]-[15], [17]. Due to this attenuation mechanism, the Empirical CDFs of OV MV BPL topologies, which are demonstrated in Figs. 4(a)-(d), normally start to increase from zero whereas Empirical CDF of UN MV BPL topologies, which are presented in Figs. 5(a)-(d), start to increase from positive values greater than zero depending on the examined indicative UN MV BPL topology. Also, apart from the distribution BPL rural case, Empirical CDFs of OV MV BPL topologies reach up to 1 at significant higher coupling scheme channel attenuation differences in contrast with the CDFs of UN MV BPL topologies.

- In the vast majority of the indicative distribution BPL topologies examined, Gaussian, Lognormal, Wald, Weibull and Gumbel channel attenuation statistical distributions asymptotically approach heights of 0 and 1 out of the range of minimum and maximum coupling scheme channel attenuation difference of the examined BPL topology. Conversely, the Empirical channel attenuation statistical distribution accurately represents the coupling scheme channel attenuation difference for given distribution BPL topology. For that reason, horizontal steps occur in the Empirical channel attenuation statistical distribution whose extent depends on the examined distribution BPL topology. Distribution BPL topologies that are characterized by frequent and of the same height coupling scheme channel attenuation difference notches (e.g., indicative OV MV BPL rural case) tend to be characterized by CDFs of important horizontal steps.

- Since shorter horizontal steps occur in indicative UN MV BPL topologies, the CDFs of the five channel attenuation statistical distributions of the initial statistical hybrid model can better fit the CDF of the Empirical distribution of the modified statistical hybrid model in contrast with their fit in indicative OV MV BPL topologies. Despite the fact of the better fit in indicative UN MV BPL topologies, the five channel attenuation statistical distributions fail to accurately approach the $\mathrm{CDF}$ heights of 0 and 1 thus entailing that the random number generator module of Phase D is going to produce: (i) lower coupling scheme channel attenuation differences than the real ones and a lot of zero coupling scheme channel attenuation differences; and (ii) higher coupling scheme channel attenuation differences than the real ones, respectively, for given distribution BPL topology.

- The CDF of the Wald channel attenuation statistical distribution fails to fit the Empirical distribution in the indicative OV MV BPL urban case A, suburban case and rural case. This is due to the MLEs provided by the MLE computation module of Phase $\mathrm{C}$ for the given coupling scheme channel attenuation differences (see Table 1 of [6]).

- Although the random number generator module of Phase D is going to produce random coupling scheme channel attenuation differences for the distribution BPL topology members that are more realistic to the indicative ones when the Empirical channel attenuation statistical distribution is applied, this does not 
automatically imply that the Empirical distribution can achieve better capacity metrics than the Gaussian, Lognormal, Wald, Weibull and Gumbel channel attenuation statistical distributions. In accordance with [6], the performance metrics, which are used in this paper during the comparison evaluation of the initial and modified statistical hybrid model, are the capacity percentage change and average absolute capacity percentage change whose values also depend on the positions of the random coupling scheme channel attenuation differences, EMI policy and coupling scheme.

Consequently, after Phases A-D of either the initial or the modified statistical hybrid model, each BPL topology class is enriched with 100 topology members per each channel attenuation statistical distribution through the random coupling scheme channel attenuation differences that are produced by the random number generator (see in [5], [6]) for given power grid type and coupling scheme. Then, Phase E determines the coupling scheme transfer function of the members of each BPL topology class for given power grid type and coupling scheme. In the following subsections, the maximum, the average and the minimum capacity of each BPL topology class, which are hereafter denoted as capacity range of each BPL topology class, are going to be computed by the Phase F while the critical performance metrics of the capacity percentage change and average absolute capacity percentage change are evaluated so that the success of the channel attenuation statistical distribution of the initial and modified statistical hybrid model are assessed for the default operation settings as well as a number of different scenarios.

\subsection{Initial and Modified Statistical Hybrid Model - Capacity Ranges of OV MV and UN MV BPL Classes for the Default Operation Settings}

Since coupling scheme transfer functions of each distribution BPL topology class member are well computed by Phase E of the statistical hybrid model for each channel attenuation statistical distribution for given power grid type and coupling scheme, Phase $\mathrm{F}$ of the initial or modified statistical hybrid model computes the maximum, the average and the minimum capacity of each BPL topology class among its $100 \mathrm{BPL}$ topology members for given power grid type, coupling scheme, channel attenuation statistical distribution and EMI policy.

Based on the theoretical framework of [5] and the findings of [6], the comparison of the initial and modified statistical hybrid model is going to be based on the successful capacity for given channel attenuation statistical distribution in terms of the metrics of capacity percentage change and average absolute capacity percentage change. In [6], it has been demonstrated for the initial statistical hybrid model that Weibull and Wald channel attenuation statistical distributions perform the best capacity estimations in OV MV and UN MV power grid types, respectively, regardless of the examined BPL topology class when the default operation settings are assumed. In fact, the previous observations are validated by the metrics of capacity percentage change and average absolute capacity percentage change in Table 3 of [6]. In order to evaluate the success performance of the Empirical channel attenuation statistical distribution, in Fig. 6(a), the maximum, average and minimum capacity of the typical OV MV urban topology class is plotted for the Weibull distribution of the initial statistical hybrid model and the Empirical distribution of the modified statistical hybrid model when the default operation settings are assumed. Also, the capacity of the indicative BPL topology of the 


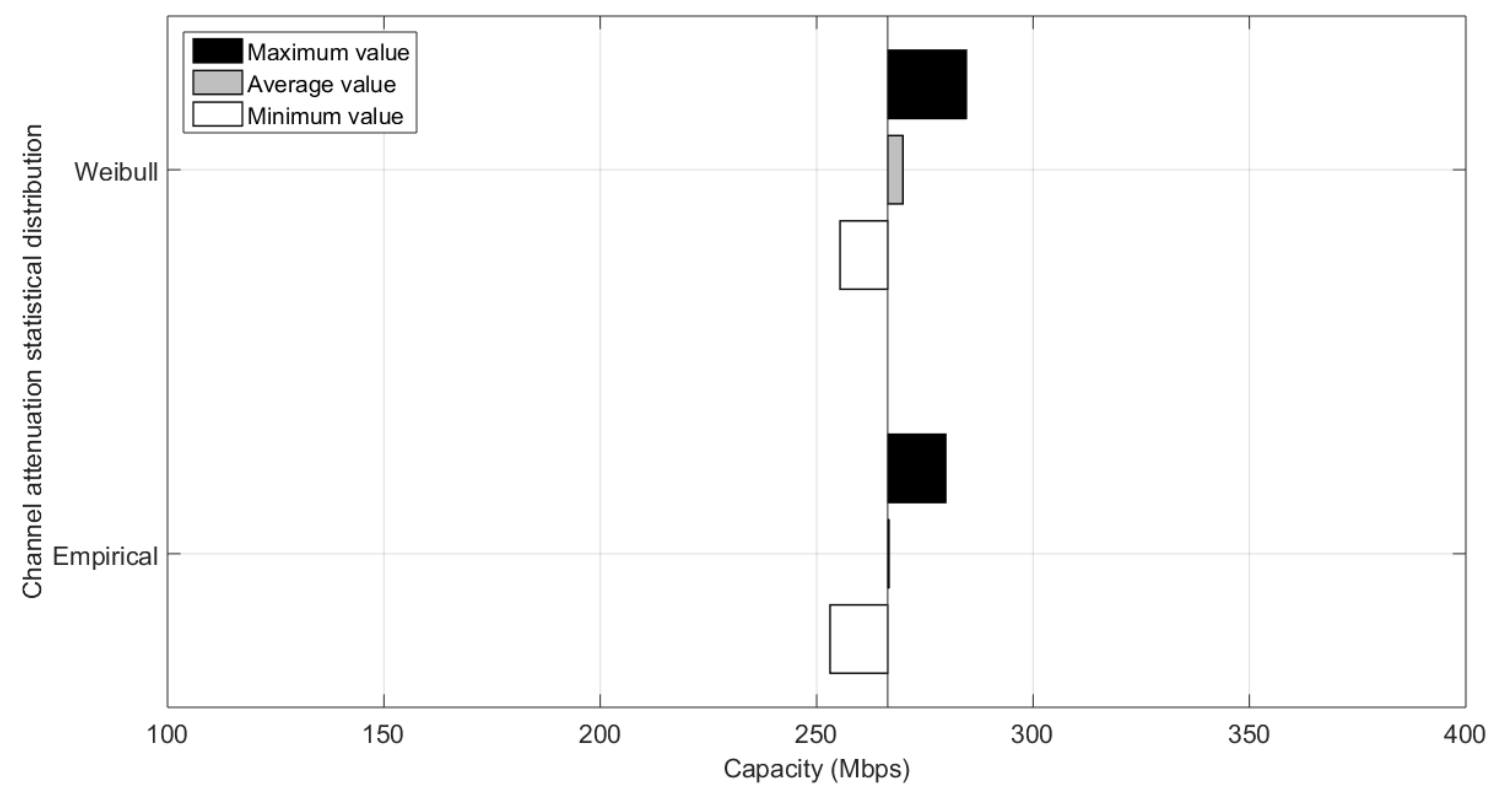

(a)

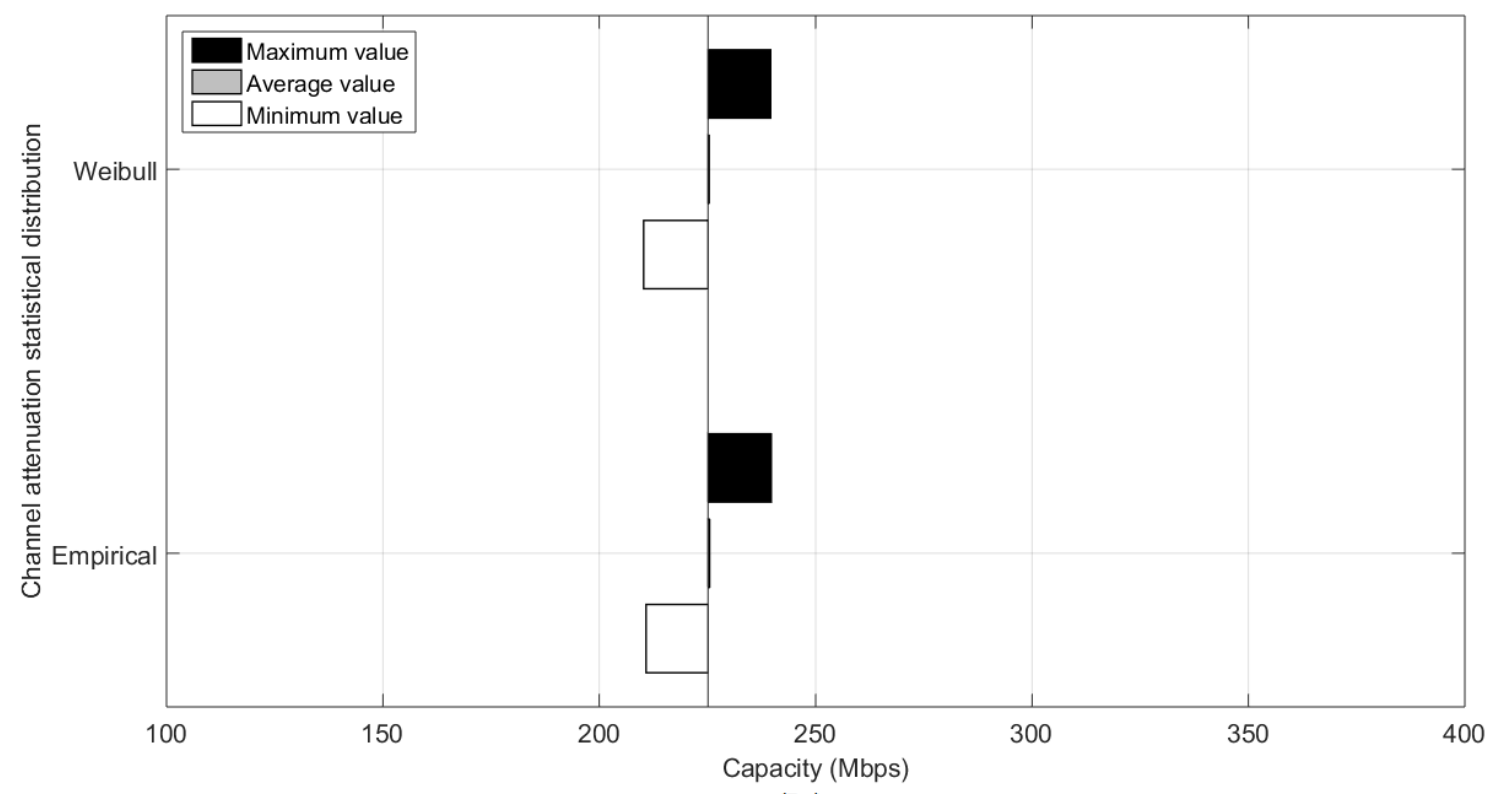

(b) 


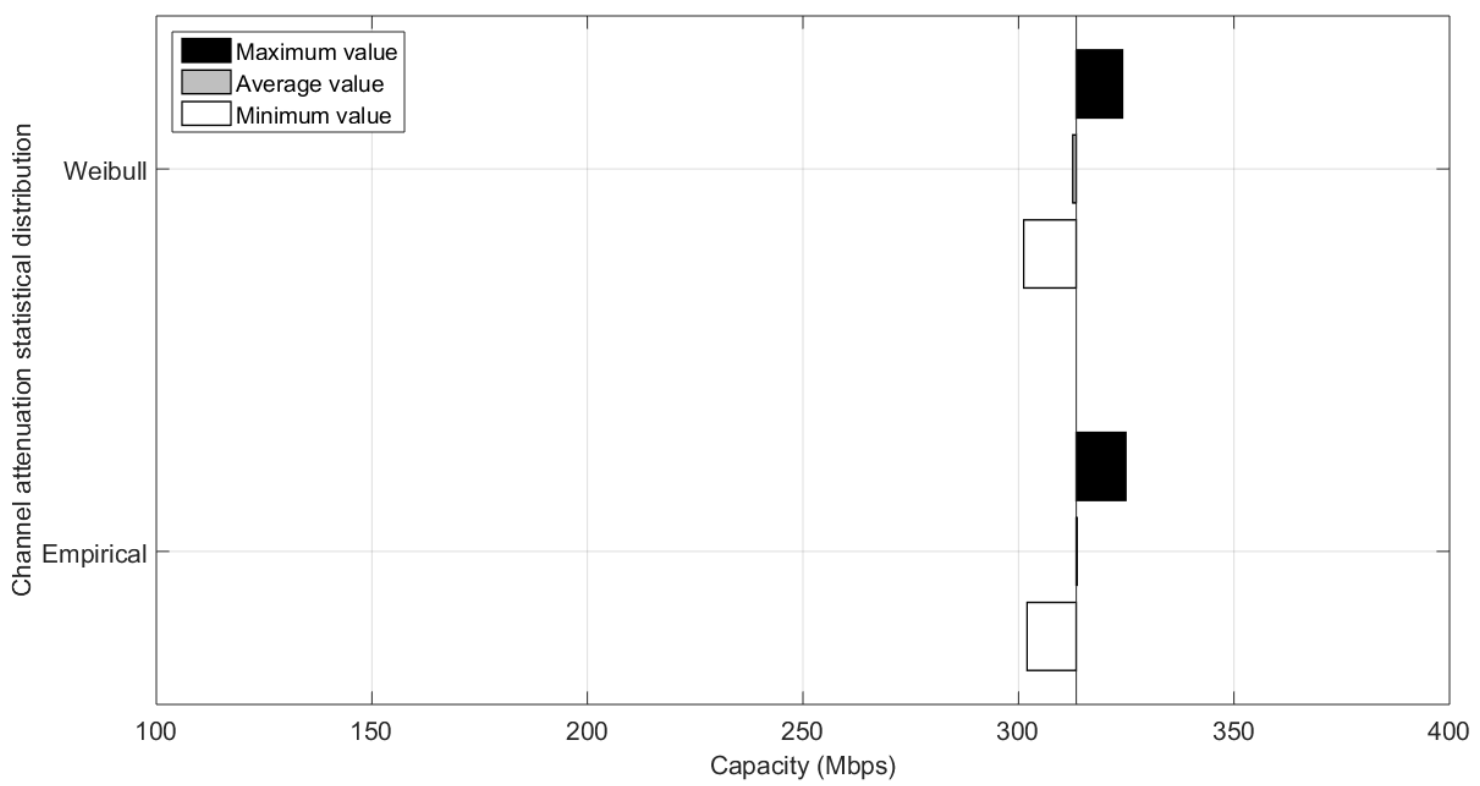

(c)

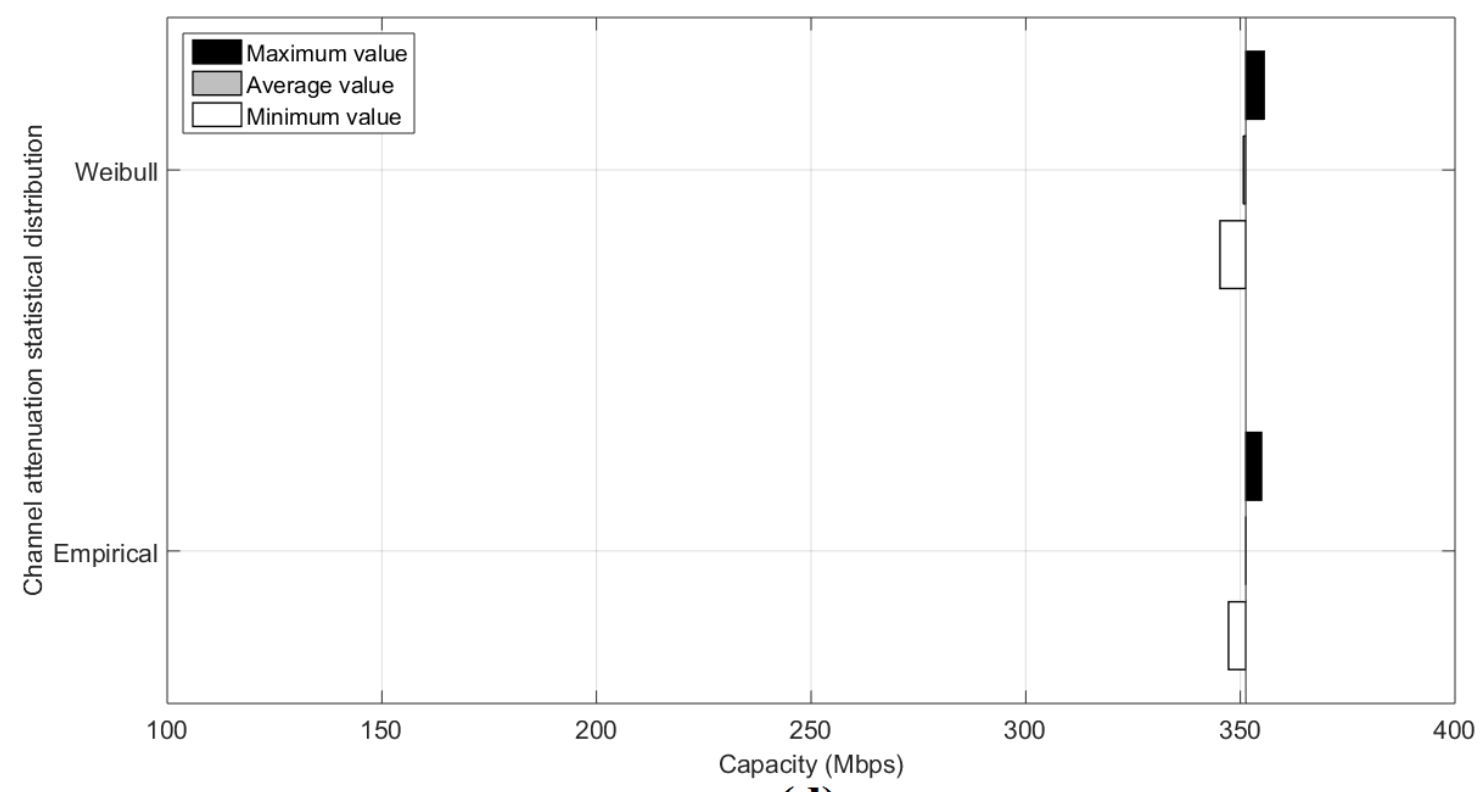

(d)

Fig. 6. Maximum, average and minimum OV MV BPL topology class capacities for the Weibull and Empirical channel attenuation statistical distribution of the initial and modified statistical hybrid model, respectively. (a) Typical OV MV BPL urban topology class. (b) Aggravated OV MV BPL urban topology class. (c) OV MV BPL suburban topology class. (d) OV MV BPL rural topology class.

BPL topology class, say, OV MV urban case A, that is computed by the hybrid model is used as the reference value of the figure. In Figs. 6(b)-(d), same bar graphs are demonstrated with Fig. 6(a) but for the aggravated OV MV BPL urban topology class, OV MV BPL suburban topology class and OV MV BPL rural topology class, respectively. Figs. 7(a)-(d) are the same with the respective Figs. 6(a)-(d) but for the UN MV BPL topology classes and Wald channel attenuation statistical distribution instead of Weibull one. 


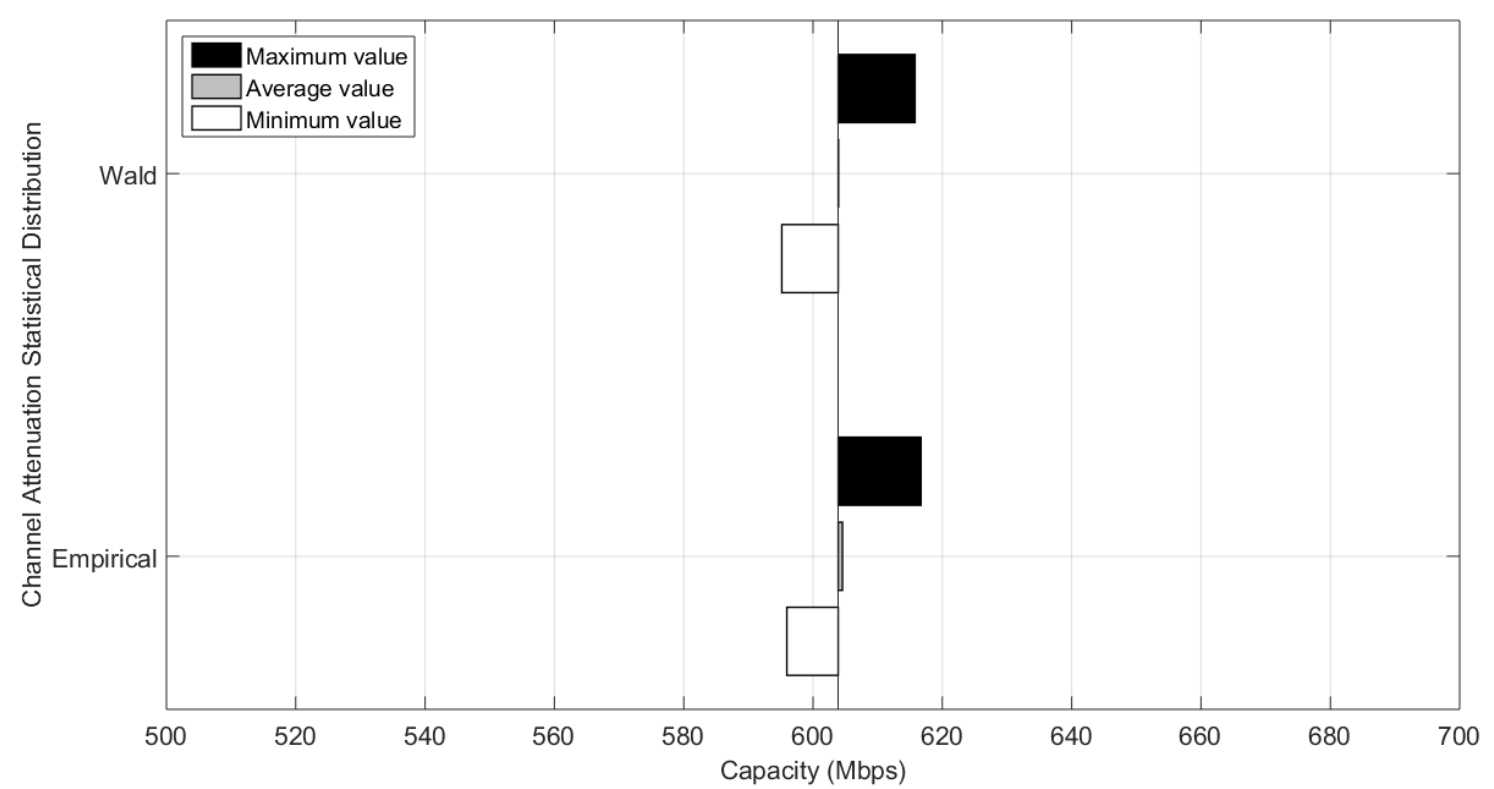

(a)

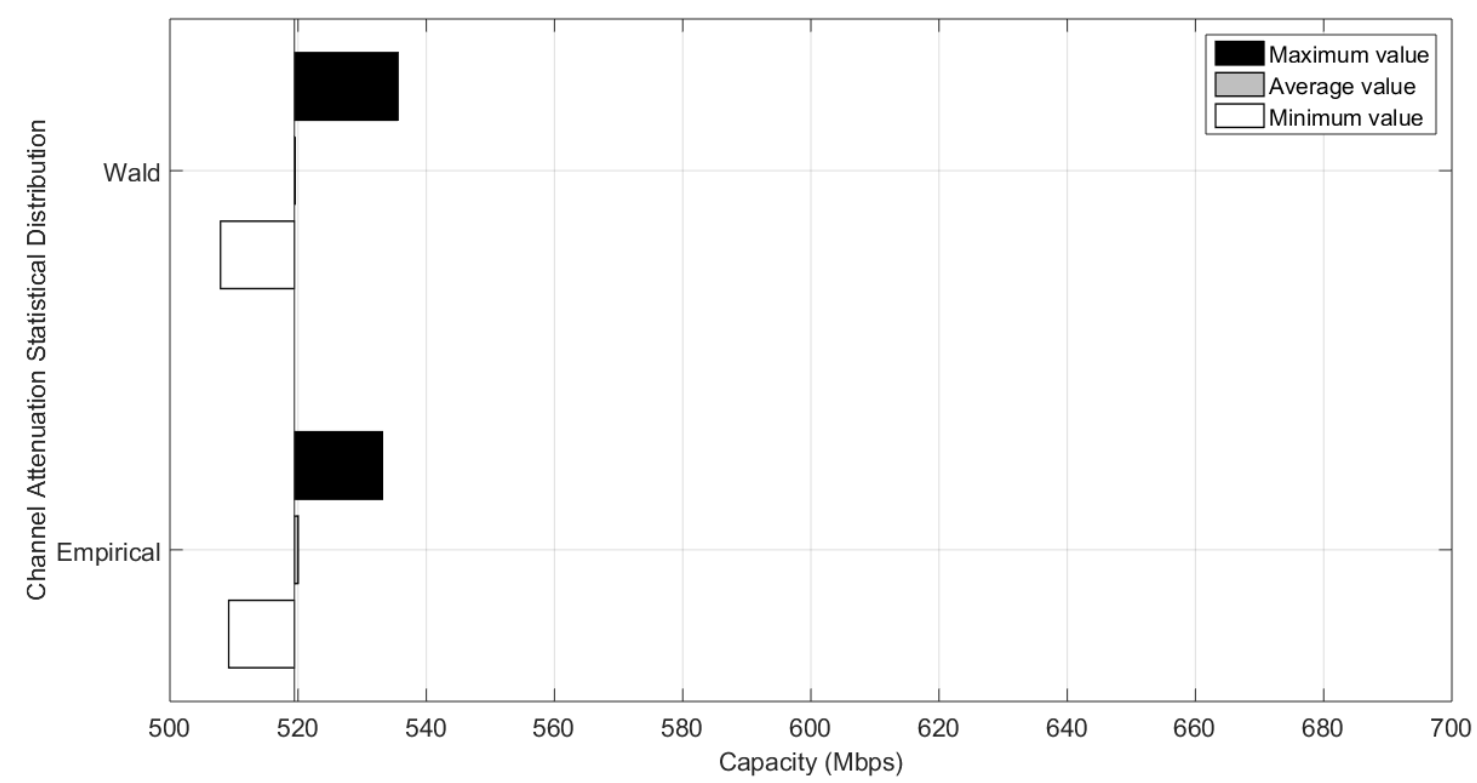

(b) 


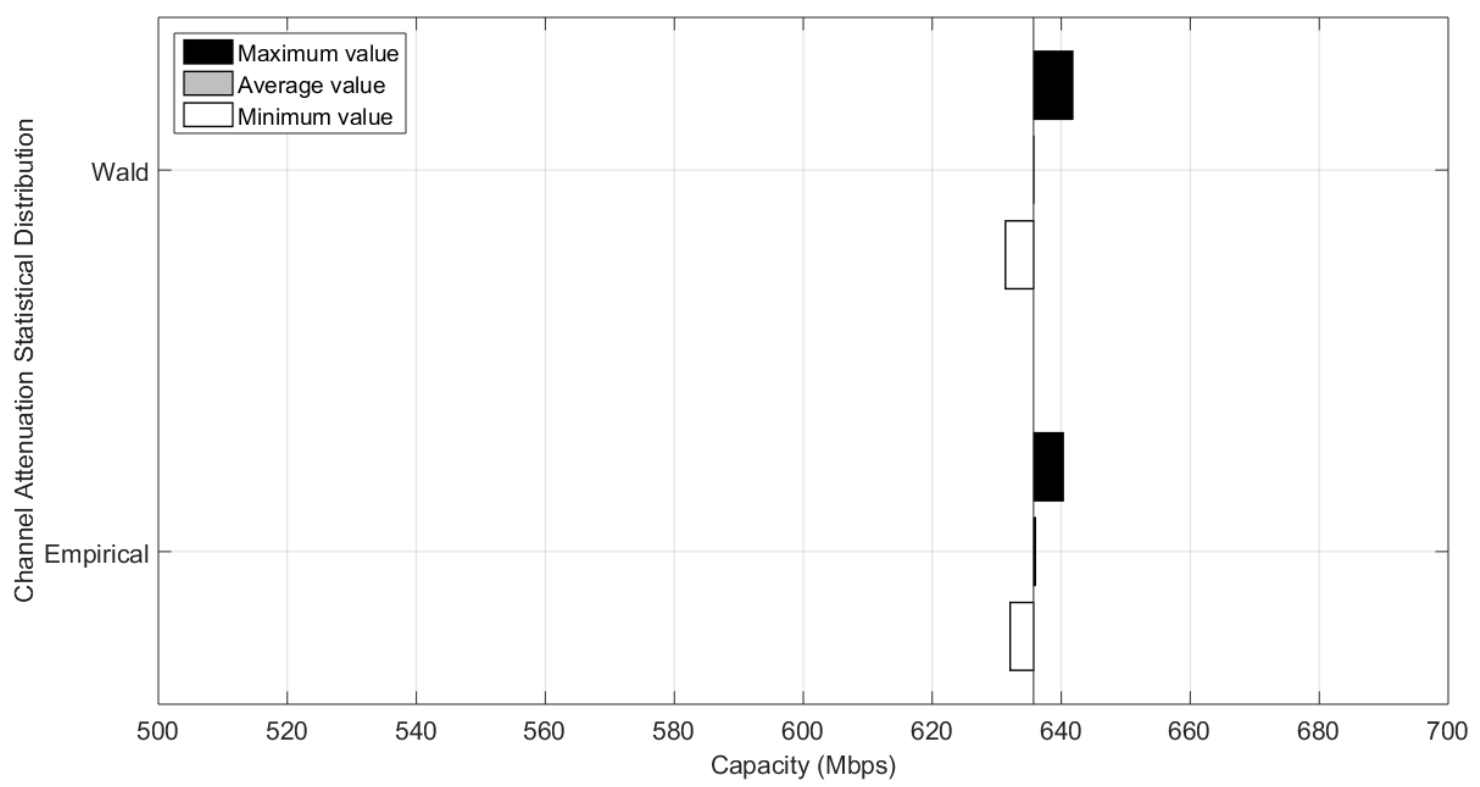

(c)

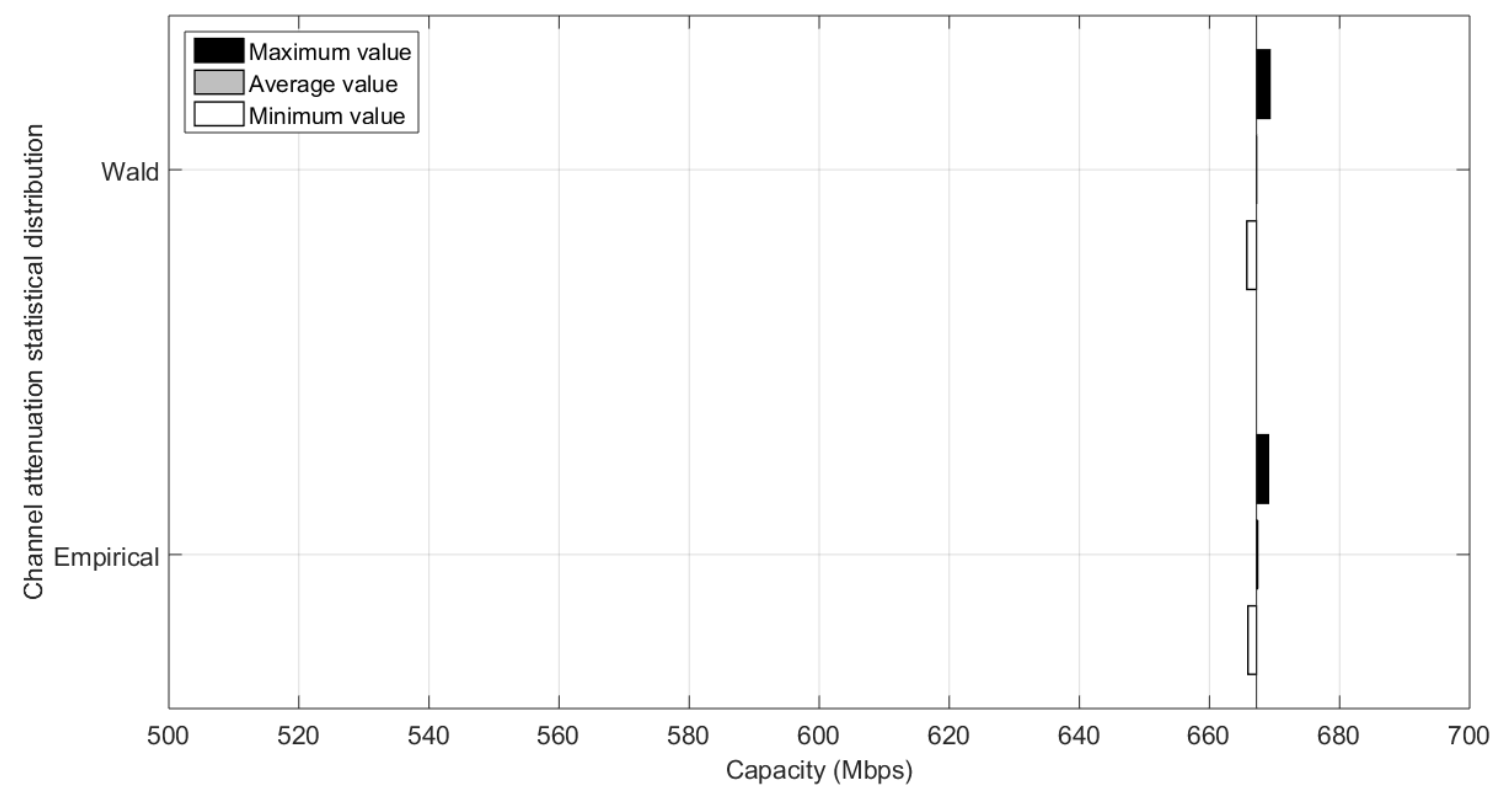

(d)

Fig. 7. Same curves with Fig. 6 but for the UN MV BPL topology classes and the Wald channel attenuation statistical distribution instead of the Weibull one .

The behavior of channel attenuation statistical distribution CDFs of Figs. 4(a)-(d) and 5(a)-(d) can be reflected on the respective Figs. 6(a)-(d) and 7(a)-(d), namely:

- In accordance with [6], a channel attenuation statistical distribution is considered to be successful if the following two criteria are satisfied, namely: (i) capacity range of each distribution BPL topology class (say, the minimum and the maximum capacity value of the distribution BPL topology class) comprises the capacity of the respective indicative distribution BPL topology; and (ii) the average capacity value of the distribution BPL topology class remains very 
close to the respective one of the indicative distribution BPL topology. Already been validated for the Weibull and Wald statistical distributions, which present the best performance for the OV MV and UN MV BPL topology classes, respectively, the Empirical channel attenuation statistical distribution also satisfies both the criteria in all the examined OV MV and UN MV BPL topology classes.

- To numerically assess the capacity estimation success of channel attenuation statistical distributions of the initial and modified statistical hybrid model, the metrics of percentage change and the average absolute percentage change, which have been proposed in [6], are applied apart from the graphical comparison of Figs. 6 and 7. Here, it should be reminded that the percentage change computes the difference percentage between the capacity average value of the examined distribution BPL topology class in relation with the respective indicative distribution BPL topology for given channel attenuation statistical distribution. Then, the average absolute percentage change is equal to the average value of the absolute percentage changes of the four distribution BPL topology classes for given power grid type and channel attenuation statistical distribution.

- In accordance with [6], high values of percentage change indicate that the estimation of the applied channel attenuation statistical distribution is unsuccessful while a threshold of 3\% (i.e., first rule of thumb in [6]) has been proposed either in the percentage change or in the average absolute percentage change so that the partial and overall success capacity estimation can be numerically checked. With reference to Table 3 of [6], it is also numerically validated that Weibull and Wald channel attenuation statistical distributions perform the best capacity estimations in OV MV and UN MV power grid types, respectively, regardless of the examined BPL topology class when the default operation settings are assumed.

In Table 3, the percentage change of each OV MV BPL topology class is reported for the Weibull channel attenuation statistical distribution, which performs the best capacity estimation in OV MV BPL topology classes for the initial statistical hybrid model, and for the Empirical channel attenuation statistical distribution for the modified statistical hybrid model. Similarly to the OV MV BPL topology class case, the percentage change of each UN MV BPL topology class is reported in the same Table but for the Wald channel attenuation statistical distribution, which performs the best capacity estimation in UN MV BPL topology classes for the initial statistical hybrid model. Also, at the bottom of the Table, the average absolute percentage change of each channel attenuation statistical distribution is given per each power grid type.

The results that are reported in Table 3 numerically verify the graphical observations of Figs. 6 and 7. More specifically:

- The rule of thumb of $3 \%$ threshold, denoted as first rule of thumb, is valid in all the cases examined for the applied channel attenuation statistical distributions when the default operation settings are assumed. The first rule of thumb is valid for the Empirical channel attenuation statistical distribution either in all distribution BPL topology classes (i.e., the absolute value of percentage change significantly remains below $3 \%$ in all the examined distribution BPL topology classes) or in the examined power grid type as a whole 
Table 3

Percentage Change of the Distribution BPL Topology Classes for the Best Channel Attenuation Statistical Distributions of the Initial Statistical Hybrid Model and the Empirical Channel Attenuation Statistical Distribution of the Modified Statistical Hybrid Model (The Default Settings Are Assumed, say,

$\mathrm{WtG}^{1} / \mathrm{StP}^{1}$ coupling scheme and FCC Part 15)

(black background: unsuccessful CDF estimation)

\begin{tabular}{|c|c|c|c|c|c|}
\hline \multirow{4}{*}{$\begin{array}{c}\text { Indicative BPL } \\
\text { Topology Name } \\
\text { (OV MV Capacity / } \\
\text { UN MV Capacity } \\
\text { in Mbps) }\end{array}$} & \multirow{4}{*}{$\begin{array}{c}\text { BPL } \\
\text { Topology } \\
\text { Class } \\
\text { Description }\end{array}$} & \multicolumn{4}{|c|}{$\begin{array}{c}\text { Percentage Change } \\
(\%)\end{array}$} \\
\hline & & \multirow{2}{*}{\multicolumn{2}{|c|}{$\begin{array}{c}\text { Initial Statistical Hybrid Model } \\
\text { Best Channel Attenuation } \\
\text { Statistical Distribution }\end{array}$}} & \multirow{2}{*}{\multicolumn{2}{|c|}{$\begin{array}{c}\text { Modified Statistical Hybrid Model } \\
\text { Empirical Channel Attenuation } \\
\text { Statistical Distribution }\end{array}$}} \\
\hline & & & & & \\
\hline & & $\begin{array}{l}\text { OV MV } \\
\text { (Weibull) }\end{array}$ & $\begin{array}{l}\text { UN MV } \\
\text { (Wald) }\end{array}$ & OV MV & UN MV \\
\hline $\begin{array}{l}\text { Urban case B } \\
(225 / 519)\end{array}$ & $\begin{array}{l}\text { Aggravated } \\
\text { BPL urban }\end{array}$ & 0.12 & 0.02 & 0.18 & 0.11 \\
\hline $\begin{array}{c}\text { Suburban case } \\
(313 / 636)\end{array}$ & $\begin{array}{c}\text { BPL suburban } \\
\text { class }\end{array}$ & -0.26 & 0.003 & 0.07 & 0.04 \\
\hline $\begin{array}{l}\text { Rural case } \\
(351 / 667)\end{array}$ & $\begin{array}{l}\text { BPL rural } \\
\text { class }\end{array}$ & -0.16 & 0.0004 & 0.01 & 0.02 \\
\hline
\end{tabular}

(i.e., the average absolute percentage change of OV MV and UN MV BPL networks also remains below 3\%). In accordance with [6] and the Table 3, Weibull and Wald channel attenuation statistical distributions comply with the first rule of thumb in OV MV and UN MV BPL topology classes, respectively. In total, since all the examined cases are in compliance with the first rule of thumb, there is no cell in black background.

- In addition, the metrics of the percentage change and the average absolute percentage change assess the success of the channel attenuation statistical distributions, namely:

○ In OV MV BPL topology classes, the application of the Empirical channel attenuation statistical distribution has achieved better percentage change results in 3 out of 4 classes (i.e., typical OV MV BPL urban class, OV MV BPL suburban class and OV MV BPL rural class) with significant improvement while the percentage change difference between the Weibull and Empirical channel attenuation statistical distributions remain low in the case of the aggravated OV MV BPL class.

- In UN MV BPL topology classes, the application of the Empirical channel attenuation statistical distribution has not achieved the best percentage change results in comparison with the ones of the Wald channel attenuation statistical distribution but the differences remain low.

- The results of the average absolute percentage change verify that the Empirical channel attenuation statistical distribution can provide reliable results that are comparable to the best ones that can be achieved by the initial statistical hybrid model through its five channel attenuation statistical distribution. 
After the graphical analysis and the performance metric analysis for the default operation settings, it is shown that the Empirical channel attenuation statistical distribution of the modified statistical hybrid model can safely substitute the statistical distributions of the initial statistical hybrid model with very satisfactory results. It should be noted that the application of the Empirical channel attenuation statistical distribution allows the bypass of the comparison/selection process among the five statistical distributions that is anyway a time consuming process and imply the application of all the five statistical distributions. However, the performance of the Empirical channel attenuation statistical distributions is investigated in Sec.5.3 and Sec.5.4 when different scenarios concerning the operation scenarios are applied similarly to Sec.2.2 and Sec.2.3 of [6], respectively.

\subsection{Initial and Modified Statistical Hybrid Models for Different EMI Policies}

Already been mentioned in Sec.4.2, EMI policies regulate the EMI of BPL system operation so that OV MV and UN MV BPL networks do not become sources of EMI to other already existing wireless and telecommunications systems [8], [13], [15], [48]-[50]. Apart from the IPSD limits of the FCC Part 15, that are considered anyway among the default operation settings, respective IPSD limits of German Reg TP NB30 and the BBC/NATO Proposal, which are computed in [43], [44], [112], can be assumed to act as the different scenarios in order to examine the impact of different EMI policies on the performance of the initial and modified statistical hybrid models.

Similarly to [6], since no MTL configuration, BPL topology and coupling scheme changes occur in the examined scenario, the CDFs and the coupling scheme channel attenuation values of the channel attenuation statistical distributions of the initial and modified statistical hybrid models concerning the indicative OV MV and UN MV BPL topologies remain the same with the respective ones of Sec. 5.1 and 5.2.

To numerically assess the performance of the initial and modified statistical hybrid model when lower IPSD limits in comparison with those of FCC Part 15 ones are applied, the percentage change and the average absolute percentage change are reported for the indicative distribution BPL topology classes of Tables 1 and 2; in Tables 4 and 5, the percentage change and the average absolute percentage change are computed when German Reg TP NB30 and BBC/NATO Proposal are applied, respectively.

In accordance with [6] and by comparing Tables 4 and 5 with Table 3, it is obvious that the more EMI protective to other telecommunication services IPSD limits (i.e., German Reg TP NB30 and BBC/NATO proposal) entail significant capacity reductions and unsuccessful capacity estimations either in OV MV or in UN MV BPL topologies regardless of the applied statistical hybrid model and channel attenuation statistical distribution. The following observations can be pointed out:

- As the initial statistical hybrid model is concerned, Weibull and Wald channel attenuation statistical distributions are not the dominant distributions to estimate in total the capacity of the OV MV and UN MV BPL topology classes, respectively. A mix of different channel attenuation statistical distributions can now describe the different distribution BPL topology classes. For each distribution BPL topology class, the selection of the best channel attenuation statistical distribution in terms of the percentage change among the five ones depends on the power grid type, the BPL topology class and 
Table 4

Same with Table 3 but for German Reg TP NB30.

\begin{tabular}{|c|c|c|c|c|c|}
\hline \multirow{3}{*}{$\begin{array}{c}\text { Indicative BPL } \\
\text { Topology Name } \\
\text { (OV MV Capacity / } \\
\text { UN MV Capacity } \\
\text { in Mbps) }\end{array}$} & \multirow{3}{*}{$\begin{array}{c}\text { BPL } \\
\text { Topology } \\
\text { Class } \\
\text { Description }\end{array}$} & \multicolumn{4}{|c|}{$\begin{array}{c}\text { Percentage Change } \\
(\%)\end{array}$} \\
\hline & & \multicolumn{2}{|c|}{$\begin{array}{c}\text { Initial Statistical Hybrid Model } \\
\text { Best Channel Attenuation } \\
\text { Statistical Distribution }\end{array}$} & \multicolumn{2}{|c|}{$\begin{array}{c}\text { Modified Statistical Hybrid Model } \\
\text { Empirical Channel Attenuation } \\
\text { Statistical Distribution }\end{array}$} \\
\hline & & OV MV & UN MV & OV MV & UN MV \\
\hline $\begin{array}{c}\text { Urban case A } \\
(5.99 / 218)\end{array}$ & $\begin{array}{l}\text { Typical BPL } \\
\text { urban class }\end{array}$ & $\begin{array}{c}1.97 \\
\text { (Gaussian) }\end{array}$ & $\begin{array}{c}-0.27 \\
\text { (Weibull) }\end{array}$ & 4.99 & 0.87 \\
\hline $\begin{array}{c}\text { Urban case B } \\
(3.93 / 144)\end{array}$ & $\begin{array}{l}\text { Aggravated } \\
\text { BPL urban }\end{array}$ & $\begin{array}{c}1.61 \\
\text { (Lognormal) }\end{array}$ & $\begin{array}{c}0.09 \\
\text { (Lognormal) }\end{array}$ & -3.37 & -0.88 \\
\hline $\begin{array}{l}\text { Suburban case } \\
(10.75 / 248)\end{array}$ & $\begin{array}{l}\text { BPL suburban } \\
\text { class }\end{array}$ & $\begin{array}{c}2.38 \\
\text { (Weibull) }\end{array}$ & $\begin{array}{c}0.001 \\
\text { (Gaussian) }\end{array}$ & 0.29 & 0.12 \\
\hline $\begin{array}{c}\text { Rural case } \\
(14.88 / 280)\end{array}$ & $\begin{array}{c}\text { BPL rural } \\
\text { class }\end{array}$ & $\begin{array}{c}1.97 \\
\text { (Weibull) }\end{array}$ & $\begin{array}{c}-0.001 \\
\text { (Gaussian) }\end{array}$ & 0.50 & 0.06 \\
\hline
\end{tabular}

Table 5

Same with Table 3 but for BBC/NATO Proposal.

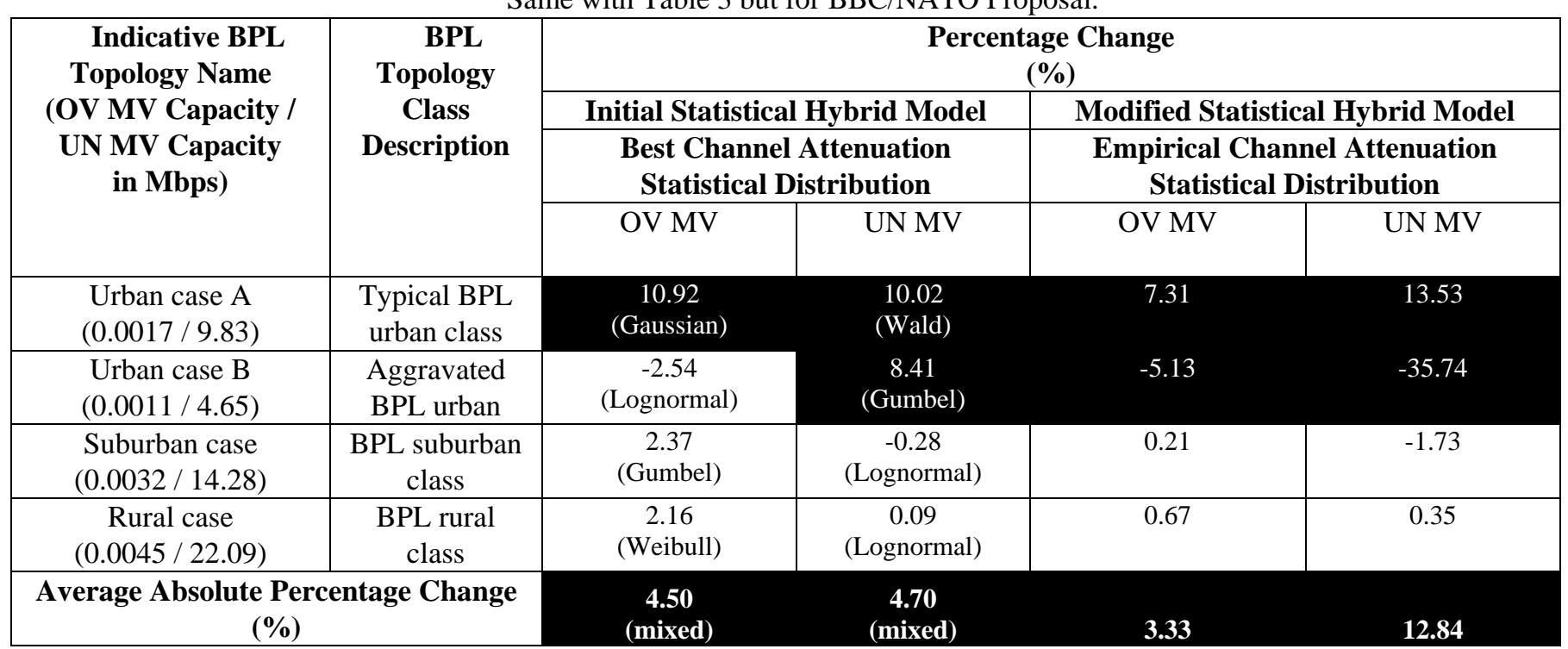

the applied EMI policy. As the modified statistical hybrid model is examined, the Empirical channel attenuation statistical distribution demonstrates a fairly stable behavior that anyway depends on the same aforementioned factors.

- As German Reg TP NB30 is applied in the indicative OV MV BPL topology classes, a mix of three different channel attenuation statistical distributions of the initial statistical hybrid model (say, Gaussian, Lognormal and Weibull) needs to be adopted so that a fine capacity estimation can be achieved in compliance with the first rule of thumb. At the same time, the Empirical channel attenuation distribution of the modified statistical hybrid model performs better estimations than the ones of the aforementioned distributions of the initial statistical hybrid model when OV MV BPL suburban and rural topology classes are examined. 
In contrast, the Empirical channel attenuation statistical distribution fails to provide a successful estimation in OV MV BPL urban topology classes.

- As BBC/NATO proposal is applied in the indicative OV MV BPL topology classes, the number of unsuccessful capacity estimations of the initial and modified statistical hybrid model skyrockets, which is anyway shown from the dramatic increase of the percentage change values. Actually, Lognormal, Gumbel and Weibull channel attenuation statistical distributions of the initial statistical hybrid model are the most suitable to estimate the capacity of the indicative OV MV BPL urban case B, suburban and rural topology classes, respectively, in compliance with the first rule of thumb while the capacity estimation of the indicative OV MV BPL urban case A topology class remains unsuccessful. Again, the Empirical channel attenuation statistical distribution of the modified statistical hybrid model achieves better capacity estimation results than the ones of the statistical distributions of the initial statistical hybrid model in the suburban and rural cases while its capacity estimation remains unsuccessful during the capacity computations of the urban cases.

- Similarly to the OV MV BPL topology classes, as German Reg TP NB30 is applied in the indicative UN MV BPL topology classes, a mix of three different channel attenuation statistical distributions of the initial statistical hybrid model (say, Weibull, Lognormal and Gaussian) needs to be implemented so that a fine capacity estimation can be achieved on the basis of the first rule of thumb. Note that the Lognormal channel attenuation statistical distribution achieves the best capacity estimations in urban case B topology class when German Reg TP NB30 is applied regardless of the examined power grid type. Here, the capacity estimations of the distributions of the initial statistical hybrid model remains better than the ones of the Empirical channel attenuation statistical distribution but with a decent percentage change difference.

- As BBC/NATO proposal is applied in the indicative UN MV BPL topology classes, the best channel attenuation statistical distribution of the initial statistical hybrid model is the Lognormal one when the suburban and rural BPL topology classes are examined. In the case of the urban BPL topology classes, the channel attenuation statistical distributions of the initial statistical hybrid model fail to perform a successful capacity estimation. In a similar way to the statistical distributions of the initial statistical hybrid model, the Empirical channel attenuation statistical distribution successfully estimates the capacity of the BPL suburban and rural topology classes in contrast with the BPL urban topology classes. Anyway, the percentage change difference between the Lognormal and the Empirical channel attenuation statistical distribution in UN MV BPL suburban and rural topology classes remains low.

- All the previous observations can be explained through the prism of the second rule of thumb proposed in [6]. In accordance with this second rule of thumb, the percentage change of the channel attenuation statistical distributions of the initial statistical hybrid model remains inversely proportional to the capacity. Indeed, when strict EMI policies are adopted, the capacity of all the indicative distribution BPL topologies is significantly reduced. Thus, the percentage changes of the channel attenuation statistical distributions of the initial statistical hybrid model increase while these percentage changes are more affected when 
$\mathrm{BBC} / \mathrm{NATO}$ proposal is adopted and urban distribution BPL topology classes are examined. The same results occur in the Empirical channel attenuation statistical distribution.

- With reference to the average absolute percentage change, the modified hybrid statistical model presents almost the same behavior with the initial one. When German Reg TP NB30 is adopted, both hybrid statistical models successfully estimate the capacities of the BPL topology classes in a whole regardless of the power grid type. Conversely, both the statistical hybrid models fail to successfully estimate the capacities of the BPL topology classes in a whole when BBC/NATO proposal is applied.

- To detect the best channel attenuation statistical distribution of the initial statistical hybrid model in terms of the percentage change, the percentage change of each statistical distribution should be computed for given distribution BPL topology class and after comparing their percentage change results, the minimum percentage change is added in Tables 3-5 for given distribution BPL topology as well as the name of the statistical distribution. Since different channel attenuation statistical distributions of the initial hybrid model are used for the different distribution BPL topology classes, the average absolute percentage change is considered as the average value of the percentage changes of the best channel attenuation statistical distributions of distribution BPL topology classes for given power grid type, coupling scheme and EMI policies. The result of the average absolute percentage change describes the best possible average absolute percentage change for the initial statistical hybrid model that is anyway unachievable by only one channel attenuation statistical distribution. Conversely, the evaluation of the percentage change of the Empirical channel attenuation statistical distribution of the modified statistical hybrid model, which is added in Tables 3-5, is a straightforward process since there is no need for comparison delays among channel attenuation statistical distributions. At the same time, the evaluation of the average absolute percentage change of the Empirical channel attenuation statistical distribution of the modified statistical hybrid model, which is added in Tables 3-5, is the average among the four percentage changes for given power grid type, coupling scheme and EMI policy, which is again an easy and straightforward computation task.

- In accordance with [6], the unsuccessful capacity estimations of the channel attenuation statistical distributions when strict EMI policies are adopted is due to the fact that the German Reg TP NB30 and BBC/NATO proposal EMI policies present a strong frequency selective behavior [24]. Hence, the random position of the random coupling scheme channel attenuation differences produced by the random number generator module of Phase D is the suspect of the unsuccessful capacity estimations rather CDFs of random number generator.

- A trade-off between the computation / comparison time and the capacity estimation accuracy is here defined for the UN MV BPL topology classes. The initial statistical hybrid model achieves a slight improved percentage change and average absolute percentage change results in UN MV BPL topology classes in comparison with the ones of the modified statistical hybrid model regardless of the applied EMI policies but the computational time of the initial statistical hybrid model remains significantly higher in comparison with the one of the modified 
statistical hybrid model. In contrast, when OV MV BPL topology classes are examined, the Empirical channel attenuation statistical distribution achieves significantly better percentage change and average absolute percentage change results in comparison with the ones of the five channel attenuation statistical distributions of the initial statistical hybrid model. At the same time, the Empirical channel attenuation statistical distribution achieves significantly better computational times. As the OV MV BPL topology classes are concerned, the modified statistical hybrid model is a straightforward selection.

In accordance with [6], strict EMI policies undermine the capacity estimations of the channel attenuation statistical distributions. Taking into account the high comparison delays of the initial statistical hybrid model, the Empirical channel attenuation statistical distribution of the modified statistical hybrid model proposes a fair and fast capacity estimation. Also, when OV MV BPL topology classes are examined, the Empirical channel attenuation statistical distributions offers the best possible and rapid capacity estimation. Apart from the different ISPD limits, the capacity estimation performance of the Empirical channel attenuation statistical distributions should also be examined when different coupling schemes are applied.

\subsection{Initial and Modified Statistical Hybrid Models for Different Coupling Schemes}

Already detailed in [5], [28], [47], CS2 module, which is the most updated BPL coupling scheme module, describes the injection of the input BPL signal onto and the extraction of the output BPL signal from the power lines of BPL networks through three different types of coupling schemes, namely: (1) Coupling Scheme Type 1: Wire-to-Ground $(W t G)$ or Shield-to-Phase $(S t P)$ coupling schemes for OV or UN BPL networks, respectively; (2) Coupling Scheme Type 2: Wire-to-Wire (WtW) or Phase-to-Phase (PtP) coupling schemes for OV or UN BPL networks, respectively; and (3) Coupling Scheme Type 3: MultiWire-to-MultiWire (MtM) or MultiPhase-toMultiPhase (MtM) coupling schemes for OV or UN BPL networks, respectively.

In [6], one representative coupling scheme per each coupling scheme type of CS2 module and power grid type has been chosen to be presented, namely: (i) Coupling Scheme Type 1: $\mathrm{WtG}^{1}$ and $\mathrm{StP}^{1}$ coupling schemes have been chosen for the OV MV and UN MV BPL networks, respectively; (ii) Coupling Scheme Type 2: WtW ${ }^{1-2}$ and $\mathrm{PtP}^{1-2}$ coupling schemes are applied for OV MV and UN MV BPL topology classes, respectively; and (iii) Coupling Scheme Type 3: $\mathrm{MtM}_{0.8}^{1-2-3}-0.1-0.1$ coupling scheme is applied for OV MV and UN MV BPL topology classes. In this paper, the aforementioned coupling schemes are applied for comparison reasons.

As the five channel attenuation statistical distributions of the initial statistical hybrid model are concerned, the different coupling schemes entail changes in coupling scheme channel attenuation and capacity values and for that reason new MLEs for the indicative OV MV BPL topologies of Table 1 and indicative UN MV BPL topologies of Table 2 should be recomputed. In fact, the MLEs of the five channel attenuation distributions of the initial statistical hybrid model for the indicative OV MV BPL topologies are reported in Table 6 of [6] when the default operation settings are assumed and $W \mathrm{tW}^{1-2}$ and $\mathrm{MtM}_{0.8-0.1-0.1}^{1-2-3}$ coupling schemes are applied. In Table 7 of [6], MLEs of the five channel attenuation distributions of the initial statistical hybrid model for the indicative UN MV BPL topologies are reported when the default operation settings are assumed and $\mathrm{PtP}^{1-2}$ and $\mathrm{MtM}_{0.8-0.1}^{1-2-3}-0.1$ coupling schemes are applied. As 
the capacity and the corresponding metrics are regarded, the percentage change between each average capacity of the distribution BPL topology class and the capacity of the indicative topology of the respective class for the channel attenuation distributions of the initial and modified statistical hybrid model is given in Table 6 when the default operation settings are applied but for the $\mathrm{WtW}^{1-2}$ and $\mathrm{PtP}^{1-2}$ coupling schemes for $\mathrm{OV}$ MV and UN MV BPL topology classes, respectively. Here it should be noted that the respective MLEs of Table 6 and 7 of [6] for the five channel attenuation statistical distributions are applied while the best channel attenuation statistical distribution in terms of the percentage change and the Empirical channel attenuation statistical distribution are considered in the case of the initial and the modified statistical hybrid model, respectively. Similarly to Table 3, at the bottom of the Table, the average percentage change of each channel attenuation statistical distribution is given per each power grid type. In Table 7, same Table with Table 6 is given but for the application of $\mathrm{MtM}_{0.8-0.1}^{1-2-3}-0.1$ coupling scheme for OV MV and UN MV BPL topology classes.

By comparing Tables 6 and 7 with Table 3, important observations can be made concerning the capacity estimation performance from the channel attenuation statistical distributions when different coupling schemes of CS2 module are applied, namely:

- In accordance with [6] and [47], by adopting CS2 module, WtW coupling schemes of OV MV BPL topologies and PtP coupling schemes of UN MV BPL topologies become almost equivalent to respective WtG coupling schemes and StP coupling schemes in terms of channel attenuation and capacity. MtM coupling schemes can achieve slightly better channel attenuation and capacity results in comparison with the ones of the previous coupling schemes due to their adaptive use of the available conductors of the examined MTL configurations.

- The impact of the different coupling schemes on the percentage change remains significantly lower in comparison with the impact of the different EMI policies. Additional evidence of this assertion is that among the channel attenuation statistical distributions of the initial statistical hybrid model, Weibull and Wald channel attenuation statistical distributions still best estimate capacity in OV MV and UN MV BPL topology classes, respectively, regardless of the coupling scheme applied when the default operation settings are adopted. In the case of the Empirical channel attenuation statistical distribution, the strongest evidence is that the percentage change values remain extremely lower in the case of the different coupling schemes rather than in the case of the different EMI policies.

- The second rule of thumb does not occur during the application of the Empirical channel attenuation statistical distribution since the observed percentage changes of the OV and UN MV BPL topology classes remain comparable. This is due to the fact that Empirical channel attenuation statistical distribution is based on Empirical CDFs in both power grid types and not on the best CDF of channel attenuation statistical distributions of the initial statistical hybrid model in terms of the percentage change.

- Different coupling schemes have little impact on the percentage change of Empirical channel attenuation statistical distribution for given power grid type and BPL topology class. Anyway, small percentage change differences among coupling schemes can be observed when MtM coupling schemes are applied. 
Table 6

Same with Table 3 but for WtW $W^{1-2}$ and $\mathrm{PtP}^{1-2}$ coupling schemes for OV MV and UN MV BPL topology classes, respectively

\begin{tabular}{|c|c|c|c|c|c|}
\hline \multirow{4}{*}{$\begin{array}{c}\text { Indicative BPL } \\
\text { Topology Name } \\
\text { (OV MV Capacity / } \\
\text { UN MV Capacity } \\
\text { in Mbps) }\end{array}$} & \multirow{4}{*}{$\begin{array}{c}\text { BPL } \\
\text { Topology } \\
\text { Class } \\
\text { Description }\end{array}$} & \multicolumn{4}{|c|}{$\begin{array}{c}\text { Percentage Change } \\
(\%)\end{array}$} \\
\hline & & \multirow{2}{*}{\multicolumn{2}{|c|}{$\begin{array}{c}\text { Initial Statistical Hybrid Model } \\
\text { Best Channel Attenuation } \\
\text { Statistical Distribution }\end{array}$}} & \multirow{2}{*}{\multicolumn{2}{|c|}{$\begin{array}{c}\text { Modified Statistical Hybrid Mode } \\
\text { Empirical Channel Attenuation } \\
\text { Statistical Distribution }\end{array}$}} \\
\hline & & & & & \\
\hline & & $\begin{array}{c}\text { OV MV } \\
\text { (Weibull) }\end{array}$ & $\begin{array}{l}\text { UN MV } \\
\text { (Wald) }\end{array}$ & OV MV & UN MV \\
\hline $\begin{array}{c}\text { Urban case A } \\
(232 / 550)\end{array}$ & $\begin{array}{l}\text { Typical BPL } \\
\text { urban class }\end{array}$ & 1.72 & 0.01 & 0.10 & 0.12 \\
\hline $\begin{array}{l}\text { Urban case B } \\
(193 / 465)\end{array}$ & $\begin{array}{l}\text { Aggravated } \\
\text { BPL urban }\end{array}$ & 0.66 & 0.04 & 0.28 & 0.12 \\
\hline $\begin{array}{l}\text { Suburban case } \\
(278 / 582)\end{array}$ & $\begin{array}{l}\text { BPL suburban } \\
\text { class }\end{array}$ & -0.27 & 0.003 & 0.09 & 0.05 \\
\hline $\begin{array}{l}\text { Rural case } \\
(317 / 613)\end{array}$ & $\begin{array}{l}\text { BPL rural } \\
\text { class }\end{array}$ & -0.20 & 0.0005 & 0.02 & 0.03 \\
\hline \multicolumn{2}{|c|}{$\begin{array}{c}\text { Average Absolute Percentage Change } \\
(\%)\end{array}$} & 0.71 & 0.02 & 0.12 & 0.08 \\
\hline
\end{tabular}

Table 7

Same with Table 3 but for MtM $\mathrm{M}_{0.8}^{1-2-\Omega_{-} \mathbb{1}_{-}-0.1}$ coupling scheme for OV MV and UN MV BPL topology classes

\begin{tabular}{|c|c|c|c|c|c|}
\hline \multirow{4}{*}{$\begin{array}{c}\text { Indicative BPL } \\
\text { Topology Name } \\
\text { (OV MV Capacity / } \\
\text { UN MV Capacity } \\
\text { in Mbps) }\end{array}$} & \multirow{4}{*}{$\begin{array}{c}\text { BPL } \\
\text { Topology } \\
\text { Class } \\
\text { Description }\end{array}$} & \multicolumn{4}{|c|}{$\begin{array}{c}\text { Percentage Change } \\
(\%)\end{array}$} \\
\hline & & \multirow{2}{*}{\multicolumn{2}{|c|}{$\begin{array}{c}\text { Initial Statistical Hybrid Model } \\
\text { Best Channel Attenuation } \\
\text { Statistical Distribution }\end{array}$}} & \multirow{2}{*}{\multicolumn{2}{|c|}{$\begin{array}{c}\text { Modified Statistical Hybrid Model } \\
\text { Empirical Channel Attenuation } \\
\text { Statistical Distribution }\end{array}$}} \\
\hline & & & & & \\
\hline & & $\begin{array}{l}\text { OV MV } \\
\text { (Weibull) }\end{array}$ & $\begin{array}{l}\text { UN MV } \\
\text { (Wald) }\end{array}$ & OV MV & UN MV \\
\hline $\begin{array}{c}\text { Urban case A } \\
(243 / 571) \\
\end{array}$ & $\begin{array}{c}\text { Typical BPL } \\
\text { urban class } \\
\end{array}$ & 1.60 & 0.01 & 0.26 & 0.12 \\
\hline $\begin{array}{c}\text { Urban case } B \\
(203 / 487)\end{array}$ & $\begin{array}{l}\text { Aggravated } \\
\text { BPL urban }\end{array}$ & 0.34 & 0.03 & 0.51 & 0.12 \\
\hline $\begin{array}{c}\text { Suburban case } \\
(290 / 603)\end{array}$ & $\begin{array}{l}\text { BPL suburban } \\
\text { class }\end{array}$ & -0.27 & 0.003 & 0.16 & 0.04 \\
\hline $\begin{array}{l}\text { Rural case } \\
(327 / 635)\end{array}$ & $\begin{array}{l}\text { BPL rural } \\
\text { class }\end{array}$ & -0.12 & 0.0004 & 0.44 & 0.03 \\
\hline \multicolumn{2}{|c|}{$\begin{array}{c}\text { Average Absolute Percentage Change } \\
(\%)\end{array}$} & 0.58 & 0.01 & 0.34 & 0.08 \\
\hline
\end{tabular}

- Also in the case of different coupling schemes, the same trade-off relation between the computation / comparison time and the capacity estimation accuracy occurs during the selection of the initial and modified statistical hybrid model. With reference to the average absolute percentage change of Tables 3, 6 and 7, Weibull and Wald channel attenuation statistical distributions remain a permanent selection when the OV MV and UN MV BPL topology classes are examined, 
respectively, but Empirical channel attenuation statistical distribution can very satisfactorily cope with both distribution power grid types.

Concluding this Section, the main disadvantage that has been highlighted in [6] is mitigated in this paper when relatively high EMI policies occur; without estimating MLEs and without losing MLE computation and comparison time, the Empirical channel attenuation statistical distribution can successfully estimate the capacity of OV MV and UN MV BPL topology classes by achieving results that are better in at least the half of the cases in comparison with the respective results of the best channel attenuation statistical distribution of the initial statistical hybrid model. Even in the cases where the Empirical channel attenuation statistical distribution does not achieve the best capacity estimation results, the performance differences in comparison with the best channel attenuation statistical distribution remain limited.

\section{Conclusions}

In this paper, the performance results of the modified statistical hybrid model have been compared against the results of the initial statistical hybrid model when various scenarios occur such as different power grid types, BPL topology classes, EMI policies and coupling schemes. In fact, based on the benchmark of [6], the Empirical channel attenuation statistical distribution has been compared against the Gaussian, Lognormal, Wald, Weibull and Gumbel channel attenuation statistical distributions with reference to the aforementioned scenarios. With reference to the capacity metrics of percentage change and average absolute percentage change as well as the first rule of thumb, the Empirical channel attenuation statistical distribution of the modified hybrid statistical model better estimates the capacity of the OV MV BPL topology classes in comparison with the Weibull channel attenuation statistical distribution of the initial statistical hybrid model when the default operation are assumed. Also, the capacity estimation results of the Empirical channel attenuation statistical distribution of the modified hybrid statistical model remain comparable to the ones of the Wald channel attenuation statistical distribution. Note that Weibull and Wald channel attenuation statistical distributions achieve the best capacity estimation results when the initial statistical hybrid model is adopted and the default operation settings are assumed. When different EMI policies, which are more protective to the other telecommunications services that coexist with BPL systems and are characterized by relatively lower IPSD limits (e.g., German Reg TP NB30 and BBC/NATO proposal), are assumed, same results concerning the capacity estimation result comparison between the Empirical channel attenuation statistical distribution and the set of channel attenuation statistical distributions of the initial statistical hybrid model occur although the percentage change and average absolute percentage change significantly increase as IPSD limits of the examined EMI policies decrease. In addition, the same number of unsuccessful capacity estimations approximately occurs between the Empirical channel attenuation statistical distribution and the best channel attenuation statistical distribution of the initial statistical hybrid model in relation with the percentage change. In the scenario of different coupling schemes, the impact of the different coupling schemes remains negligible on the percentage change and the average absolute percentage of all the channel attenuation statistical distributions in comparison with the respective impact of the different EMI policies. Again, the performance results of the Empirical channel attenuation statistical distribution coincide with the ones of the default operation settings scenario. In addition, 
a trade-off between the computation / comparison time and the capacity estimation accuracy has been defined for the initial and modified statistical hybrid models. Hence, apart from the high capacity estimation performance of the Empirical channel attenuation statistical distribution, the modified statistical hybrid model achieves low computation time and zero comparison time since only one, say, the Empirical channel attenuation statistical distribution, occurs. Concluding this paper, the proposed modified statistical hybrid model with its Empirical channel attenuation statistical distribution offers a rapid and accurate upgrade of the initial statistical hybrid model that is considered as a valuable simulation tool towards the enrichment of BPL topology classes with realistic BPL topology members.

\section{CONFLICTS OF INTEREST}

The author declares that there is no conflict of interests regarding the publication of this paper.

\section{References}

[1] F. Xia, L. T. Yang, L. Wang, and A. Vinel, "Internet of things," International Journal of Communication Systems, vol. 25, no. 9, pp. 1101-1102, 2012.

[2] L. Atzori, A. Iera, and G. Morabito, "The Internet of Things: A survey," Elsevier Comput Netw., vol. 54, no. 15, pp. 2787-2805, 2010.

[3] H. Farhangi, "The path of the smart grid," IEEE Power and Energy Magazine, vol. 8, no. 1, pp. 18-28, 2010.

[4] A. S. de Beer, A. Sheri, H. C. Ferreira, and A. H. Vinck, "Channel frequency response for a low voltage indoor cable up to $1 \mathrm{GHz}$," In Power Line Communications and its Applications (ISPLC), 2018 IEEE International Symposium on, pp. 1-6, 2018.

[5] A. G. Lazaropoulos, "Statistical Broadband over Power Lines Channel Modeling Part 1: the Theory of the Statistical Hybrid Model," Progress In Electromagnetics Research C, vol. 92, pp. 1-16, 2019. [Online]. Available: http://www.jpier.org/PIERC/pierc92/01.19012902.pdf

[6] A. G. Lazaropoulos, "Statistical Broadband over Power Lines Channel Modeling Part 2: the Numerical Results of the Statistical Hybrid Model," Progress In Electromagnetics Research C, vol. 92, pp. 17-30, 2019. [Online]. Available: http://www.jpier.org/PIERC/pierc92/02.19012903.pdf

[7] A. G. Lazaropoulos, "Underground Distribution BPL Connections with $(\mathrm{N}+1)$ hop Repeater Systems: A Novel Capacity Mitigation Technique," Elsevier Computers and Electrical Engineering, vol. 40, pp. 1813-1826, 2014.

[8] A. G. Lazaropoulos, "Review and Progress towards the Capacity Boost of Overhead and Underground Medium-Voltage and Low-Voltage Broadband over Power Lines Networks: Cooperative Communications through Two- and ThreeHop Repeater Systems," ISRN Electronics, vol. 2013, Article ID 472190, pp. 119, 2013. [Online]. Available: http://www.hindawi.com/isrn/electronics/aip/472190/

[9] A. G. Lazaropoulos, "Broadband over Power Lines (BPL) Systems Convergence: Multiple-Input Multiple-Output (MIMO) Communications Analysis of Overhead 
and Underground Low-Voltage and Medium-Voltage BPL Networks (Invited Paper)," ISRN Power Engineering, vol. 2013, Article ID 517940, pp. 1-30, 2013. [Online]. Available: http://www.hindawi.com/isrn/power.engineering/2013/517940/

[10] A. Nazem and M. R Arshad, "An Approach in Full Duplex Digital Multipoint Systems Using Large Signal Power Line Communication," Bentham Recent Patents on Electrical \& Electronic Engineering, vol. 6, no. 2, pp. 138-146, 2013.

[11] A. G. Lazaropoulos, "Deployment Concepts for Overhead High Voltage Broadband over Power Lines Connections with Two-Hop Repeater System: Capacity Countermeasures against Aggravated Topologies and High Noise Environments," Progress in Electromagnetics Research B, vol. 44, pp. 283-307, 2012. [Online]. Available: http://www.jpier.org/PIERB/pierb44/13.12081104.pdf

[12] A. G. Lazaropoulos and P. G. Cottis, "Transmission characteristics of overhead medium voltage power line communication channels," IEEE Trans. Power Del., vol. 24, no. 3, pp. 1164-1173, Jul. 2009.

[13] A. G. Lazaropoulos and P. G. Cottis, "Capacity of overhead medium voltage power line communication channels," IEEE Trans. Power Del., vol. 25, no. 2, pp. 723-733, Apr. 2010.

[14] A. G. Lazaropoulos and P. G. Cottis, "Broadband transmission via underground medium-voltage power lines-Part I: transmission characteristics," IEEE Trans. Power Del., vol. 25, no. 4, pp. 2414-2424, Oct. 2010.

[15] A. G. Lazaropoulos and P. G. Cottis, "Broadband transmission via underground medium-voltage power lines-Part II: capacity," IEEE Trans. Power Del., vol. 25, no. 4, pp. 2425-2434, Oct. 2010.

[16] A. G. Lazaropoulos, "Broadband transmission and statistical performance properties of overhead high-voltage transmission networks," Hindawi Journal of Computer Networks and Commun., 2012, article ID 875632, 2012. [Online]. Available: http://www.hindawi.com/journals/jcnc/aip/875632/

[17] A. G. Lazaropoulos, "Towards Modal Integration of Overhead and Underground Low-Voltage and Medium-Voltage Power Line Communication Channels in the Smart Grid Landscape: Model Expansion, Broadband Signal Transmission Characteristics, and Statistical Performance Metrics (Invited Paper)," ISRN Signal Processing, vol. 2012, Article ID 121628, pp. 1-17, 2012. [Online]. Available: http://www.hindawi.com/isrn/sp/2012/121628/

[18] A. G. Lazaropoulos, "Towards Broadband over Power Lines Systems Integration: Transmission Characteristics of Underground Low-Voltage Distribution Power Lines," Progress in Electromagnetics Research B, vol. 39, pp. 89-114, 2012. [Online]. Available: http://www.jpier.org/PIERB/pierb39/05.12012409.pdf

[19] P. Amirshahi and M. Kavehrad, "High-frequency characteristics of overhead multiconductor power lines for broadband communications," IEEE J. Sel. Areas Commun., vol. 24, no. 7, pp. 1292-1303, Jul. 2006.

[20] T. Sartenaer, "Multiuser communications over frequency selective wired channels and applications to the powerline access network" Ph.D. dissertation, Univ. Catholique Louvain, Louvain-la-Neuve, Belgium, Sep. 2004.

[21] T. Calliacoudas and F. Issa, "“Multiconductor transmission lines and cables solver," An efficient simulation tool for plc channel networks development," presented at the IEEE Int. Conf. Power Line Communications and Its Applications, Athens, Greece, Mar. 2002. 
[22] T. Sartenaer and P. Delogne, "Deterministic modelling of the (Shielded) outdoor powerline channel based on the multiconductor transmission line equations," IEEE J. Sel. Areas Commun., vol. 24, no. 7, pp. 1277-1291, Jul. 2006.

[23] FCC, "In the Matter of Amendment of Part 15 regarding new requirements and measurement guidelines for Access Broadband over Power Line Systems," FCC 04-245 Report and Order, Jul. 2008.

[24] A. Milioudis, G. Andreou, and D. Labridis, "Optimum transmitted power spectral distribution for broadband power line communication systems considering electromagnetic emissions," Elsevier Electric Power Systems Research, vol. 140, pp. 958-964, 2016. DOI: 10.1016/j.epsr.2016.03.047

[25] A. G. Lazaropoulos, "A Panacea to Inherent BPL Technology Deficiencies by Deploying Broadband over Power Lines (BPL) Connections with Multi-Hop Repeater Systems," Bentham Recent Advances in Electrical \& Electronic Engineering, vol. 10, no. 1, pp. 30-46, 2017.

[26] http://matlabtricks.com/post-44/generate-random-numbers-with-a-givendistribution

[27] http://www.av8n.com/physics/arbitrary-probability.htm

[28] A. G. Lazaropoulos, "Broadband Performance Metrics and Regression Approximations of the New Coupling Schemes for Distribution Broadband over Power Lines (BPL) Networks," Trends in Renewable Energy, vol. 4, no. 1, pp. 43-73, Jan. $2018 . \quad$ [Online]. Available: http://futureenergysp.com/index.php/tre/article/view/59/pdf

[29] A. W. van der Vaart, Asymptotic Statistics. Cambridge Series in Statistical and Probabilistic Mathematics, Cambridge University Press, Cambridge, 1998. ISBN: 0-521-49603-9; 0-521-78450-6

[30] N. Suljanović, A. Mujčić, M. Zajc, and J. F. Tasič, "Approximate computation of high-frequency characteristics for power line with horizontal disposition and middle-phase to ground coupling," Elsevier Electr. Power Syst. Res., vol. 69, pp. 17-24, Jan. 2004.

[31] OPERA1, D5: Pathloss as a function of frequency, distance and network topology for various LV and MV European powerline networks. IST Integrated Project No 507667, Apr. 2005.

[32] N. Suljanović, A. Mujčić, M. Zajc, and J. F. Tasič, "High-frequency characteristics of high-voltage power line," in Proc. IEEE Int. Conf. on Computer as a Tool, Ljubljana, Slovenia, Sep. 2003, pp. 310-314.

[33] N. Suljanović, A. Mujčić, M. Zajc, and J. F. Tasič, "Power-line high-frequency characteristics: analytical formulation," in Proc. Joint 1st Workshop on Mobile Future \& Symposium on Trends in Communications, Bratislava, Slovakia, Oct. 2003, pp. 106-109.

[34] W. Villiers, J. H. Cloete, and R. Herman, "The feasibility of ampacity control on HV transmission lines using the PLC system," in Proc. IEEE Conf. Africon, George, South Africa, Oct. 2002, vol. 2, pp. 865-870.

[35] P. Amirshahi, "Broadband access and home networking through powerline networks" Ph.D. dissertation, Pennsylvania State Univ., University Park, PA, May 2006.

[36] OPERA1, D44: Report presenting the architecture of plc system, the electricity network topologies, the operating modes and the equipment over which PLC access system will be installed, IST Integr. Project No 507667, Dec. 2005. 
[37] J. Anatory, N. Theethayi, R. Thottappillil, M. M. Kissaka, and N. H. Mvungi, "The influence of load impedance, line length, and branches on underground cable Power-Line Communications (PLC) systems," IEEE Trans. Power Del., vol. 23, no. 1, pp. 180-187, Jan. 2008.

[38] J. Anatory, N. Theethayi, and R. Thottappillil, "Power-line communication channel model for interconnected networks-Part II: Multiconductor system," IEEE Trans. Power Del., vol. 24, no. 1, pp. 124-128, Jan. 2009.

[39] J. Anatory, N. Theethayi, R. Thottappillil, M. M. Kissaka, and N. H. Mvungi, "The effects of load impedance, line length, and branches in typical low-voltage channels of the BPLC systems of developing countries: transmission-line analyses," IEEE Trans. Power Del., vol. 24, no. 2, pp. 621-629, Apr. 2009.

[40] T. Banwell and S. Galli, "A novel approach to accurate modeling of the indoor power line channel-Part I: Circuit analysis and companion model," IEEE Trans. Power Del., vol. 20, no. 2, pp. 655-663, Apr. 2005.

[41] W. Villiers, J. H. Cloete, L. M. Wedepohl, and A. Burger, "Real-time sag monitoring system for high-voltage overhead transmission lines based on powerline carrier signal behavior," IEEE Trans. Power Del., vol. 23, no. 1, pp. 389-395, Jan. 2008.

[42] NTIA, "Potential interference from broadband over power line (BPL) systems to federal government radio communications at 1.7-80 MHz Phase 1 Study Vol. 1," NTIA Rep. 04-413, Apr. 2004.

[43] M. Gebhardt, F. Weinmann, and K. Dostert, "Physical and regulatory constraints for communication over the power supply grid," IEEE Commun. Mag., vol. 41, no. 5, pp. 84-90, May 2003.

[44] NATO, "HF Interference, Procedures and Tools (Interférences HF, procédures et outils) Final Report of NATO RTO Information Systems Technology," RTO-TRISTR-050, Jun. 2007.

[45] A. G. Lazaropoulos, "The Impact of Noise Models on Capacity Performance of Distribution Broadband over Power Lines Networks," Hindawi Computer Networks and Communications, vol. 2016, Article ID 5680850, 14 pages, 2016. doi:10.1155/2016/5680850. [Online]. Available: http://www.hindawi.com/journals/jenc/2016/5680850/

[46] A. G. Lazaropoulos, "Capacity Performance of Overhead Transmission MultipleInput Multiple-Output Broadband over Power Lines Networks: The Insidious Effect of Noise and the Role of Noise Models (Invited Paper)," Trends in Renewable Energy, vol. 2, no. 2, pp. 61-82, Jun. $2016 . \quad$ [Online]. Available: http://futureenergysp.com/index.php/tre/article/view/23

[47] A. G. Lazaropoulos, "New Coupling Schemes for Distribution Broadband over Power Lines (BPL) Networks," Progress in Electromagnetics Research B, vol. 71, pp. 39-54, 2016. [Online]. Available: http://www.jpier.org/PIERB/pierb71/02.16081503.pdf

[48] A. G. Lazaropoulos, "Designing Broadband over Power Lines Networks Using the Techno-Economic Pedagogical (TEP) Method-Part I: Overhead High Voltage Networks and Their Capacity Characteristics (Invited Review Article)," Trends in Renewable Energy, vol. 1, no. 1, pp. 16-42, Mar. 2015. [Online]. Available: http://futureenergysp.com/index.php/tre/article/view/2 
[49] A. G. Lazaropoulos, "Overhead and Underground MIMO Low Voltage Broadband over Power Lines Networks and EMI Regulations: Towards Greener Capacity Performances," Elsevier Computers and Electrical Engineering, vol. 39, pp. 2214-2230, 2013.

[50] D. Fenton and P. Brown, "Some aspects of benchmarking high frequency radiated emissions from wireline communications systems in the near and far fields," in Proc. IEEE Int. Symp. on Power Line Communications and its Applications, Malmö, Sweden, Apr. 2001, pp. 161-167.

Article copyright: (C) 2019 Athanasios G. Lazaropoulos. This is an open access article distributed under the terms of the Creative Commons Attribution 4.0 International License, which permits unrestricted use and distribution provided the original author and source are credited. 